\title{
The Solar Activity Monitor Network - SAMNet
}

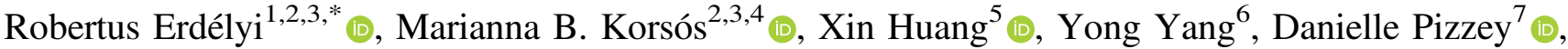
Steven A. Wrathmall ${ }^{7}$ (1), Ifan G. Hughes ${ }^{7}$ (1), Martin J. Dyer ${ }^{8}$ (1), Vikram S. Dhillon ${ }^{8,9}$ (D), Bernadett Belucz ${ }^{2,3}$, Roman Brajša ${ }^{10}$, Piyali Chatterjee ${ }^{11}$, Xuewu Cheng ${ }^{6}$, Yuanyong Deng ${ }^{5}$, Santiago Vargas Domínguez ${ }^{12}$ (D), Raúl Joya ${ }^{13}$ (D), Peter Gömöry ${ }^{14}$ (D), Norbert G. Gyenge ${ }^{1,3}$, Arnold Hanslmeier ${ }^{15}$, Ales Kucera ${ }^{14}$, David Kuridze ${ }^{4,16}$, Faquan Li ${ }^{6}$, Zhong Liu ${ }^{17,18}$, Long Xu ${ }^{5}$ (D), Mihalis Mathioudakis ${ }^{19}$, Sarah Matthews ${ }^{20}$ (D), James R.T. McAteer ${ }^{21,22}$ (D), Alexei A. Pevtsov ${ }^{23,24}$ (1), Werner Pötzi $^{25}$ (1) , Paolo Romano ${ }^{26}$ (D), Jinhua Shen ${ }^{27}$, János Temesváry ${ }^{3}$, Andrey G. Tlatov ${ }^{28}$ (D), Charles Triana $^{29}$ (D), Dominik Utz ${ }^{15,30}$, Astrid M. Veronig ${ }^{15,25}$ (D), Yuming Wang ${ }^{31,32}$, Yihua Yan ${ }^{5,33}$ (D), Teimuraz Zaqarashvili ${ }^{15,16,34}$, and Francesca Zuccarello ${ }^{35}$

${ }^{1}$ Solar Physics \& Space Plasma Research Center (SP2RC), School of Mathematics and Statistics, University of Sheffield, Hicks Building, Hounsfield Road, Sheffield S3 7RH, UK

2 Department of Astronomy, Eötvös Loránd Univ., Pázmány P. Sétány 1/A, Budapest 1117, Hungary

${ }^{3}$ Gyula Bay Zoltán Solar Observatory (GSO), Hungarian Solar Physics Foundation (HSPF), Petöfi tér 3., Gyula 5700, Hungary

${ }^{4}$ Department of Physics, Aberystwyth University, Ceredigion, Cymru SY23 3BZ, UK

${ }^{5}$ CAS Key Laboratory of Solar Activity, National Astronomical Observatories, Chinese Academy of Sciences, A20 Datun Rd. Chaoyang District, Beijing 100012, PR China

${ }^{6}$ State Key Laboratory of Magnetic Resonance and Atomic and Molecular Physics, Innovation Academy for Precision Measurement Science and Technology, Chinese Academy of Science (CAS), West No. 30 Xiao Hong Shan, Wuhan 430071, PR China

7 Joint Quantum Centre (JQC) Durham-Newcastle, Department of Physics, Durham University, South Road, Durham DH1 3LE, UK

${ }^{8}$ Department of Physics and Astronomy, University of Sheffield, Sheffield S3 7RH, UK

9 Instituto de Astrofísica de Canarias, La Laguna, Tenerife 38205, Spain

${ }^{10}$ Hvar Observatory, Faculty of Geodesy, Univ. of Zagreb, Kačićeva 26, Zagreb 10000, Croatia

11 Indian Institute of Astrophysics (IIA), 2nd Block Koramangala, Bangalore 560034, India

12 Observatorio Astronómico Nacional, Universidad Nacional de Colombia, Av. Carrera 30 \# 45-03, Bogotá, Colombia

13 Observatorio Astronómico, Universidad Sergio Arboleda, Av. Carrera 30 \# 45-03, Bogotá, Colombia

14 Astronomical Institute, Slovak Academy of Sciences, Tatranská Lomnica 05960, Slovakia

15 Institute of Physics, University of Graz, Universitätsplatz 5, Graz 8010, Austria

16 Abastumani Astrophysical Observatory, Mount Kanobili 383762, Georgia

17 Yunnan Observatories, Chinese Academy of Sciences, Kunming 650011, PR China

${ }^{18}$ School of Astronomy and Space Science, Univ. of CAS, Beijing 100049, PR China

19 Astrophysics Research Centre, School of Mathematics and Physics, Queen's University Belfast, Belfast BT7 1NN, UK

${ }^{20}$ UCL-Mullard Space Science Lab., Holmbury St Mary, Dorking, Surrey RH5 6NT, UK

${ }^{21}$ Department of Astronomy, New Mexico State Univ, P.O. Box 30001, MSC 4500, Las Cruces, NM 88003, USA

22 Sunspot Solar Observatory, Sunspot, NM 88349, USA

${ }^{23}$ National Solar Observatory, Boulder, CO 80303, USA

${ }^{24}$ Pulkovo Astronomical Observatory, Russian Academy of Sciences, Saint Petersburg 196140, Russian Federation

${ }^{25}$ Kanzelhöhe Observatory for Solar and Environmental Research, University of Graz, Kanzelhöhe 19, Treffen 9521, Austria

${ }^{26}$ INAF Osservatorio Astrofisico di Catania, Via S. Sofia 78, Catania 95123, Italy

27 Xinjiang Astromomical Observatory, CAS, 150 Science 1-Street, Urumqi 830011, PR China

${ }^{28}$ Kislovodsk Solar Mountain Astronomical Station, Central Astronomical Observatory of the Russian Academy of Sciences, Kislovodsk 357700, Russia

29 Observatorio AstroExplor, Cra 58C 152B-22, Bogota, Colombia

${ }^{30}$ Instituto de Astrofsica de Andaluca, Granada 18008, Spain

${ }^{31}$ CAS Key Laboratory of Geospace Environment, School of Earth and Space Sciences, Univesity of Science and Technology of China, Hefei 230026, PR China

32 CAS Center for Excellence in Comparative Planetology, USTC, Hefei 230026, PR China

33 School of Astronomy and Space Science, Univ. of Chinese Acad. of Sciences, Beijing 100049, PR China

${ }^{34}$ School of Natural Sciences and Medicine, Ilia State Univ., Cholokashvili Ave. 3/5, Tbilisi 0162, Georgia

35 Dipartimento di Fisica e Astronomia "Ettore Majorana", Universitá di Catania, Via S. Sofia 78, 95123 Catania, Italy

\footnotetext{
*Corresponding author: robertus@sheeld.ac.uk
} 
Received 16 June 2020 / Accepted 10 June 2021

\begin{abstract}
The Solar Activity Magnetic Monitor (SAMM) Network (SAMNet) is a future UK-led international network of ground-based solar telescope stations. SAMNet, at its full capacity, will continuously monitor the Sun's intensity, magnetic, and Doppler velocity fields at multiple heights in the solar atmosphere (from photosphere to upper chromosphere). Each SAMM sentinel will be equipped with a cluster of identical telescopes each with a different magneto-optical filter (MOFs) to take observations in $\mathrm{K} \mathrm{I}$, Na D, and Ca I spectral bands. A subset of SAMM stations will have white-light coronagraphs and emission line coronal spectropolarimeters. The objectives of SAMNet are to provide observational data for space weather research and forecast. The goal is to achieve an operationally sufficient lead time of e.g., flare warning of 2-8 h and provide many sought-after continuous synoptic maps (e.g., LoS magnetic and velocity fields, intensity) of the lower solar atmosphere with a spatial resolution limited only by seeing or diffraction limit, and with a cadence of $10 \mathrm{~min}$. The individual SAMM sentinels will be connected to their master HQ hub where data received from all the slave stations will be automatically processed and flare warning issued up to $26 \mathrm{~h}$ in advance.
\end{abstract}

Keywords: Sun-flares-precursors

\section{Introduction}

The Earth's atmosphere and the magnetosphere are constantly buffeted by a continuous flow of solar particles originating in the solar atmosphere. This continuous flow may suddenly be enhanced and enter into the Earth's upper atmosphere when a high-intensity solar flare (e.g., above M5-class, using the GOES classification) or a massive coronal mass ejection (CME) erupts in the solar atmosphere and reaches Earth after propagating across the interplanetary space. The frequency of these most energetic events of the entire Solar System follows the 11-year solar cycle.

At the peak of the solar activity cycle, several dangerously high-intensity class flares and CMEs may occur (on average, around 2-3 per day at maximum). When a CME associated with a flare arrives at Earth several days later, its interaction with Earth's magnetosphere may trigger geomagnetic storms, with the additional (sometimes dramatic) consequences for various technological systems critical to our civilization (e.g., natural gas and oil pipelines, power lines, telecommunication, navigation, etc). These societal assets and services are vital for the economic welfare and security of every citizen but may be subject to failure due to solar activity, as it has indeed happened in the past (see e.g., Eastwood et al., 2017).

A crucial difference between today's world and the one from many centuries ago is that we are now substantially more reliant upon computers and communications to run our commerce, work facilities, and even our forms of entertainment and recreation. The initial 15 geostationary and a few tens of Molniya-orbit communication satellites operating in 1989 have grown to more than several hundred by now. Space weather may now affect such critical areas as (1) the communication and navigation systems, (2) spacecraft operations, (3) global technological systems: power line distribution, pipeline operations, geophysical exploration, (4) research: balloon and rocket experiments, carbon dating, ozone measurements, and (5) the aircraft and commercial space travel (see, Fig. 1 in Pevtsov, 2017). Our current economic development places even greater demands on the power network infrastructure and near-Earth space-based assets. Due to such a reliance on global technological systems, it is now believed that one of the biggest threats of civilisation breakdown may come from space weather.
Therefore the question is not any more "whether" it will occur, but "when" and "how strong". A direct consequence of and follow-up to this question is that one should improve space weather early warning system by developing new capabilities for the observations and modelling of space weather precursors. This latter is the context of this current paper. With the Solar Activity Monitor Network (SAMNet) we now take a step in the direction to develop a ground-based facility for advanced space weather warning, in particular, to improve flare forecasting. The core scientific concept and underpinning scientific justification (see Sect. 2) behind SAMNet is the idea to exploit the line-of-sight (LoS) component primarily of the magnetic and additionally of the velocity (i.e., Doppler) field observable not just at the visible surface of the Sun (i.e., in the photosphere) but at a range of heights in the lower solar atmosphere to improve the lead time for flare warning.

In this paper, we focus on the use of the measurable LoS component of magnetic data as this is a more popular and developed area for space weather pre-cursor applications. However, there is emerging literature about how the Doppler data of the near-side Sun may be used for imaging the emergence of the active region of the far-side (see e.g., Zhao et al., 2019). Another growing application of the Doppler velocity fields is related to the evolution of magnetic helicity. In particular, the shearing and emerging components of helocity may provide an additional and complementary pre-flare warning. How to determine these helicity components of the temporally varying magnetic helicity, how to assess their capability to serve as flare warning, and how information on the LoS velocity components of the magnetised plasma will constrain the analysis is demonstrated e.g., by Ye et al. (2018) and Korsós et al. (2020b).

Novel flare prediction methods recently developed have already demonstrated, through observations and 3D simulations of active region (AR) dynamics, that information about the magnetic field at various heights in the lower solar atmosphere, in particular in the chromosphere, has the potential to considerably improve forecasting lead times. Therefore, a facility is now needed to provide a routine and continuous supply of magnetic and velocity field data of the entire solar disk, not just ARs, at various atmospheric heights and in particular the chromosphere. One needs to emphasise at this point that SAMNet and its core 
(a)
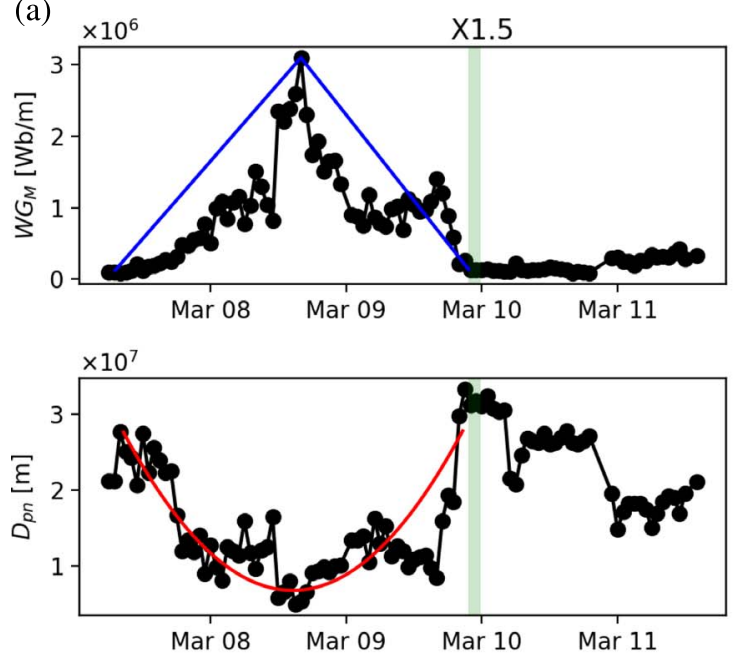

(b)
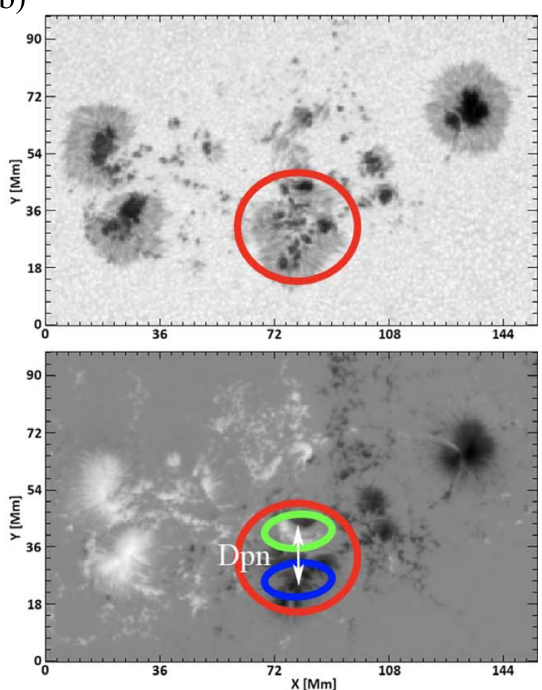

Fig. 1. Diagnostic diagrams of a sample investigated AR 11166. The upper panel of column (a) is the variation of the $W_{\mathrm{M}}$ parameter and the lower panel shows the evolution of the distance $\left(D_{p n}\right)$. The right panels in column (b) are the white light (upper panel) and LoS magnetogram (lower panel) images of AR 11166 taken at 07:59 on 9 March 2011.

Solar Activity Magnetic Monitor (SAMM) instrument will be capable of acquiring the full disk magnetograms, which then can be used for a more in-depth analysis of sub-areas around ARs.

After a summary in Section 2.1, the so-called weighted magnetic gradient $\left(\mathrm{WG}_{\mathrm{M}}\right)$ method is introduced. $\mathrm{WG}_{\mathrm{M}}$ is particularly developed to exploit measurements of a SAMNet-type facility. The method is based on assessing the evolution of the horizontal gradient of LoS component of magnetic fields observable by a series of SAMMs, the core instruments of SAMNet, in focused areas of ARs. Although we demonstrate the underlying science for ARs only, one has to recognise the importance of full-disk magnetic observations. For example, CMEs often originate from (and their magnetic field is defined by) the chromospheric filament eruptions. Observing only ARs may miss other source regions of CMEs. Also, CME triggers may be remote, and so, without a global (full disk) view they could be missed too. In Section 2.2, arguments are also made using machine learning in favour of why a cost-effective set of lowresolution diffraction-limited ground-based solar telescope systems like SAMNet may provide data with sufficient resolution and accuracy for flare eruption warning. In Section 2.3, it is demonstrated what gains in forecasting lead time may be achieved by applying the $\mathrm{WG}_{\mathrm{M}}$ method to SAMM-type LoS magnetic data of the lower solar atmosphere. Here, the concept of optimum height is introduced where such LoS data of ARs may yield the best lead time (see Sect. 2.3.3). Next, in Section 3, the concept of MOF is introduced with applications to observe the lower solar atmosphere, followed by Section 4 devoted to the novel idea of tuning MOFs. Section 5 embarks on the introduction of the SAMM, an instrument realisation of mounting MOF-based filters on solar telescopes. The Solar Activity Monitor Network as an ensemble of SAMM solar telescope stations is introduced and discussed in Section 6. Finally, Section 7 discusses the potential future directions and limitations of a SAMNet-type of the flare forecasting system.

\section{Forecasting using LoS component of solar magnetic fields: $W_{M}$ method and $M L$ approach as underpinning science}

Here, we embark on a brief but necessary scientific justification in favour of the $W_{\mathrm{M}}$ method that enables e.g., flare forecasting at source, using LoS solar atmospheric magnetic data that are obtained by e.g., a series of SAMM sentinels of SAMNet. In the Introduction, it was mentioned that we employ ARs as examples. However, the method may work equally well for the entire solar disk, subject to some limiting selection criteria outlined below (e.g., for reducing inaccuracy caused by measuring the magnetic field close to limb, etc.). It is also not claimed that the $\mathrm{WG}_{\mathrm{M}}$ method is superior when compared to other methods. There are several excellent methods available in the literature that on a range of predictive parameters of solar eruptions (see e.g., Georgoulis, 2013; Barnes et al., 2016; Leka et al., 2018, 2019a, b, and references therein). However, most flare and CME forecast methods apply only photospheric magnetic and Doppler data of ARs for forecasting. Some recent, pioneering approaches with varying degrees of success attempt already to incorporate solar atmospheric extreme ultraviolet (EUV) data and/or use Machine Learning to improve forecasting accuracy (see e.g., Qahwaji \& Colak, 2007; Bobra \& Couvidat, 2015; Anastasiadis et al., 2017; Florios et al., 2018; Campi et al., 2019; Camporeale, 2019; Kim et al., 2019; Wang et al., 2019). Caveats of applying ML techniques are also addressed in Liu et al. (2021).

Detailed information on measuring, and the consequent modelling, of the 3D magnetic field structure of the solar magnetic field, in particular for an AR, would be important to obtain more accurate insight into the pre-flare evolution locally in the solar atmosphere. However, direct routine observations of the 3D magnetic field in the lower solar atmosphere, above the photosphere up into the top of the chromosphere, are currently 
not available. An overwhelming majority of observations these days refer to either the LoS component or the full magnetic field vector in the photosphere only. Nowadays, approximate methods for modelling the local magnetic field vector in the solar atmosphere include its construction using current free (potential, PF) or nonlinear force-free field (NLFFF) extrapolation techniques. In practice, however, to construct an accurate and reliable 3D magnetic field structure, also called the magnetic skeleton, of an AR from photospheric measurements is still a challenging task with several caveats, see Wiegelmann \& Sakurai (2012).

The focus of this section is to demonstrate why it is sufficient for an efficient and accurate flare forecasting to observe (i) only the line-of-sight ( $\mathrm{LoS})$ component of the solar magnetic field $B_{\mathrm{LoS}}$, and (ii) $B_{\mathrm{LoS}}$ at a range of heights in the lower solar atmosphere measured e.g., by an instrumented suit like SAMMs of SAMNet. In Section 2.1, the core principles and applicability of the $\mathrm{WG}_{\mathrm{M}}$ are outlined, while the section on employing machine learning (Sect. 2.2) is a key aspect to demonstrate that the skill score is not changing drastically by binning the HMI data. This latter information is in support of applying lowresolution, basically seeing-limited (and also diffraction-limited) LoS magnetic data.

\subsection{The $W G_{M}$ method}

It is shown by e.g., Schrijver (2007) that the most intense flares are in connection with the location of the strongest magnetic gradient. The weighted horizontal magnetic gradient $\left(\mathrm{WG}_{\mathrm{M}}\right)$ method was developed and introduced originally by Korsós et al. (2015, 2019, 2020a) for the pre-flare tracking of ARs in the photosphere. The concept of the method is based on the evolution of the weighted horizontal magnetic gradient of the LoS component of the magnetic field $\left(\mathrm{WG}_{\mathrm{M}}\right)$ between two opposite polarities in a $\delta$-spot at the solar surface. Therefore, one needs to identify the $\delta$-spot(s) of the selected ARs for the $\mathrm{WG}_{\mathrm{M}}$ method. To find a $\delta$-spot, we adopted and apply the automatic PIL recognition program developed by Cui et al. (2006).

Figure 1a illustrates the methodological framework of the $\mathrm{WG}_{\mathrm{M}}$ method applied to an arbitrary example, say AR11166. The $\mathrm{WG}_{\mathrm{M}}$ quantity itself is defined as a proxy to measure the magnetic non-potentiality in a contoured-up region of interest where $D_{p n}$ is the distance between the area-weighted centers of the positive and negative polarities (barycenters) in this cluster, see Figure $1 \mathrm{~b}$.

AR 11166 produced a strong X1-class flare according to the GOES flare classification system. The pre-flare dynamics and the related physical processes at the solar surface were investigated using data with an hourly temporal resolution from joint HMIDD ground- (Debrecen Heliophysical Observatory, DHO) and space-based (Helioseismic and Magnetic Imager on-board Solar Dynamics Observatory, SDO/HMI) sunspot data catalogues (see, e.g., Baranyi et al., 2016) from 2010 to 2014. The $\mathrm{WG}_{\mathrm{M}}$ analyses focused on the vicinity of polarity inversion line(s) (PILs), which is within the red ellipse(s) in Figure $1 \mathrm{~b}$. This is the area where the indicative characteristic features of the imminent flaring behaviour appear up to 2-3 days before the actual flare onset.

By inspecting Figure 1a, two important findings of the $\mathrm{WG}_{\mathrm{M}}$ method are as follows: (i) First, a prominent and characteristic pre-flare pattern of the $\mathrm{WG}_{\mathrm{M}}$ proxy quantity is identified: increasing phase, maximum and gradual decrease prior to flaring. The overall pre-flare behaviour of the $\mathrm{WG}_{\mathrm{M}}$ parameter is approximated by aqua "inverted V-shape" on the top panel of Figure 1a. This characteristic pre-flare behaviour is also confirmed by Korsós et al. $(2015,2019)$ in further approximately 130 flare cases between 1996 and 2014, where a linear relationship was found between the pre-flare $W_{M}$ maximum $\left(\mathrm{WG}_{\mathrm{M}}^{\max }\right)$ and the largest flare intensity class of the AR investigated. This relationship yields a tool to estimate the expected flare intensity following the preceding maximum of $\mathrm{WG}_{\mathrm{M}}$. (ii) Further, the introduction of $\mathrm{WG}_{\mathrm{M}}$ enables to determine a second flare precursor. Namely, the two barycenters of opposite polarities display a unique characteristic pattern of converging and diverging motions prior to the flare, which is illustrated with a red "U"-shaped parabolic curve in the lower panel of Figure 1a. This "U"-shaped precursor allows to estimate the expected onset time of flare in a $\delta$-type AR.

In summary, applying the $\mathrm{WG}_{\mathrm{M}}$-method to photospheric data only yields an about $7 \mathrm{~h}$ gain in lead time. Here, at this point, we wish to emphasise that achieving this progress is made by employing a relatively low spatial resolution (of the order of an arcsec) that is typical for ground-based observatories, as would be expected from e.g., SAMNet as a lower limit, routinely monitoring the solar surface with a cadence of as low as about an hour. SAMNet will have, however, a better cadence at about 10 mins for a fixed wavelength that enables then a more accurate flare warning.

\subsection{Application of ML to LoS magnetic fields}

In this section, we support the efforts that rely on how robust space weather forecasting, using LoS magnetic data, can be against resolution issues, i.e., how suitable a ground-based seeing-limited instrumentation is. We show that downgrading the HMI data does not influence the skills cores/probabilities of forecasting.

ML methods can be used to build a solar flare forecasting model (Camporeale, 2019). Some of these rely on parameters calculated from the observational images (Nishizuka et al., 2018; Wang et al., 2020); others directly input solar images and automatically extract forecasting patterns (Huang et al., 2018; Park et al., 2018). We apply convolutional neural networks $(\mathrm{CNN})$, which can process images directly, to explore the influence of image resolution on the performance of the solar flare forecasting model. $\mathrm{CNN}$ is mainly composed of the convolutional layer, the nonlinear layer, the pooling layer, and the fully connected layer. The convolutional layer is applied to extract forecasting patterns, the nonlinear layer provides the nonlinear transform in the forecasting model, the pooling layer is used to reduce the complexity of the forecasting model, and the fully connected layer is the last in CNN, the relationships between forecasting patterns and solar flares are obtained in this layer.

The original HMI magnetograms of active regions and their downgrading images are fed into the CNNs, respectively, as shown in Figure 2. The models can provide forecasting results by using magnetograms with different resolutions. By comparing the performances of these models, we can estimate the influence of image resolution on the forecasting model. Using bi-cubic interpolation method, we now downgrade the original image $1 \alpha(\alpha=10,20, \ldots, 100)$ times. The flaring sample is 


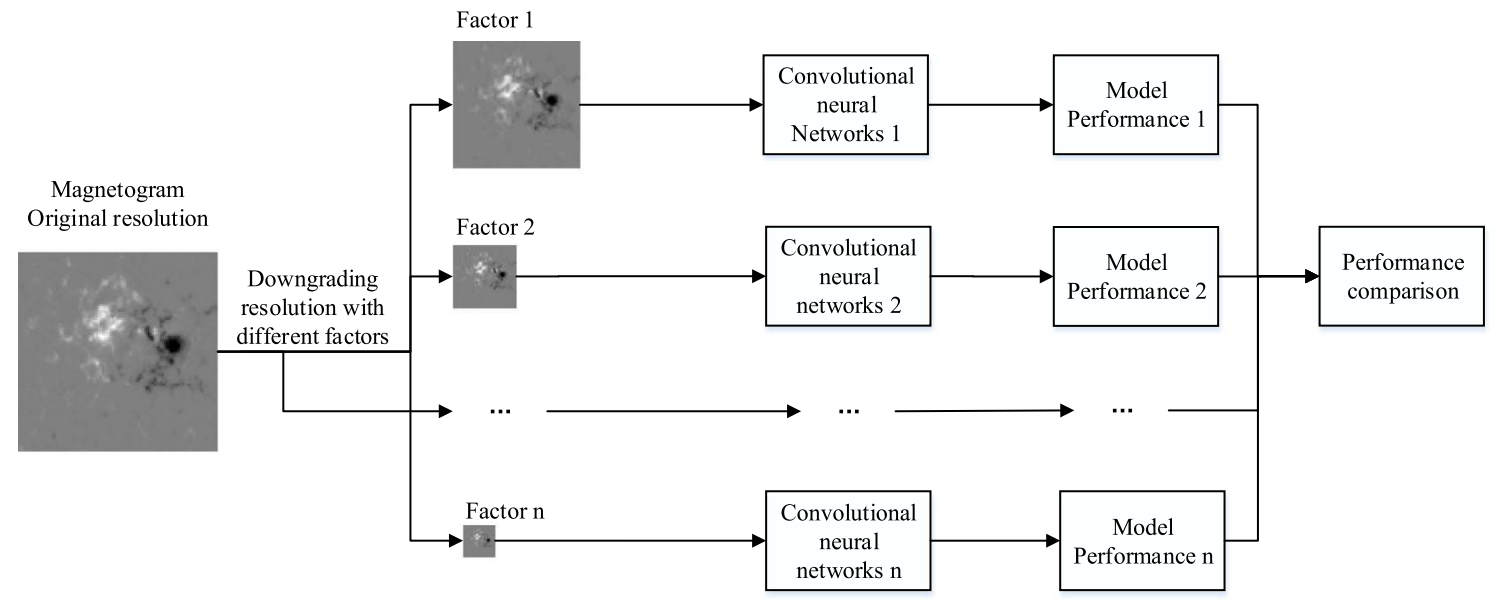

Fig. 2. Flow chart for exploring influence of resolution on model performance.

considered a positive sample, meanwhile, the non-flaring sample is considered a negative sample. Hence, there are four possible outcomes in the solar flare forecasting model: i) true positive (TP) are that samples are correctly predicted as positive; ii) true negative $(\mathrm{TN})$ are that samples are correctly predicted as negative; iii) false positive (FP) are that samples are wrongly predicted as positive; and iv) false negative $(\mathrm{FN})$ are that samples are wrongly predicted as negative.

Based on these possible outcomes, the true skill score (TSS) (Eq. (1)) is considered as the index to evaluate the model performance:

$$
\mathrm{TSS}=\frac{\mathrm{TP}}{\mathrm{TP}+\mathrm{FN}}+\frac{\mathrm{TN}}{\mathrm{TN}+\mathrm{FP}}-1 .
$$

The performance of forecasting models built on magnetograms with different spatial resolutions is shown in Figure 3. The result indicates that the ML-based forecasting model is not sensitive to the spatial resolution of the input images. The ML-based model can work well, even if the input image is downgraded to 1/90 of the original size for HMI magnetograms. This result is rather important for facilities like SAMNet that may not achieve very high resolution due to the limiting effects of seeing (and diffraction, unless going for large apertures, say above $30 \mathrm{~cm}$ or even larger).

\subsection{The power of $W G_{M}$ - Forecasting as function of height with SAMNet-type data}

Let us apply the $\mathrm{WG}_{\mathrm{M}}$ method to LoS magnetic data in the lower solar atmosphere to establish how to further increase the flare prediction capability of the method. We now describe and demonstrate with a typical example how the forecast lead time gained by applying the $\mathrm{WG}_{\mathrm{M}^{-}}$method to photospheric magnetic data can be even further improved by applying it to 3D LoS magnetic data at a range of heights across the lower solar atmosphere that may be delivered by a SAMM instrument.

For demonstrating the power of applying LoS magnetic data at a range of heights, a 3D magnetic map data catalogue is constructed, which includes the area, mean magnetic field data, and

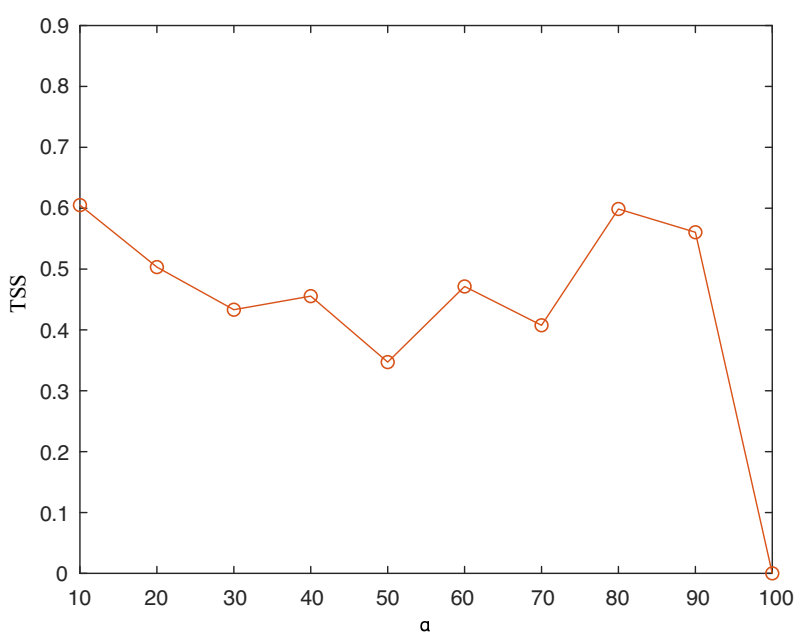

Fig. 3. Performance of forecasting models trained on magnetograms with different spatial resolutions. Original images are downgraded $1 / \alpha$ times.

the location (Carrington coordinates, $L$ for longitude, and $B$ for latitude) of sunspots of ARs at every $45 \mathrm{~km}$ in height from the photosphere into the lower corona on hourly bases for the selected ARs. Eligible ARs now have to satisfy the suit of selection criteria, see Korsós et al. (2019, 2020a). These criteria are a little different, i.e., more restrictive, from those when compared to the criteria of the photospheric event selection, applied in Section 2.1.

Once the above selection criteria are satisfied for an AR, first we use extrapolation to construct the 3D magnetic skeleton of this AR. Next, as a function of height, the $\mathrm{WG}_{\mathrm{M}^{-} \text {method is }}$ employed and the gain in the lead time of flare onset forecasting is determined and compared to the counterpart lead time using photospheric data only. Finally, we search for a range of heights where the best lead-time improvement can be achieved. Such height will be called the optimum height. Once a solar magnetic monitoring system, e.g., SAMNet, would observe the LoS component of the magnetic field at the optimum heights in 
(a)

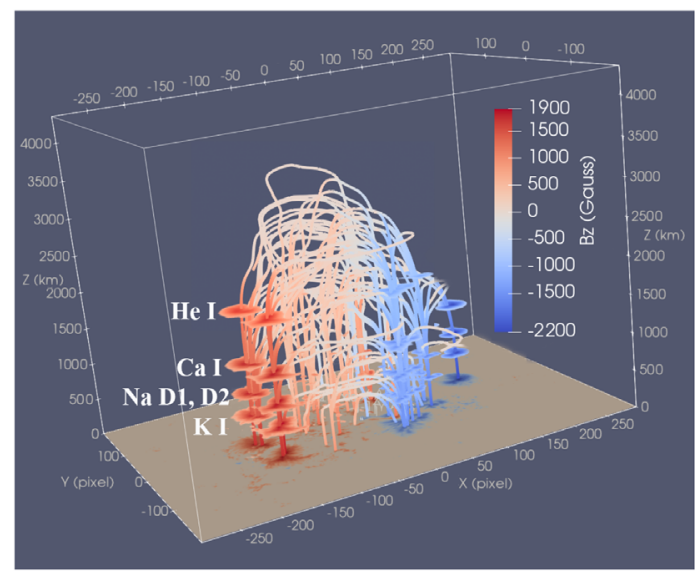

(b)

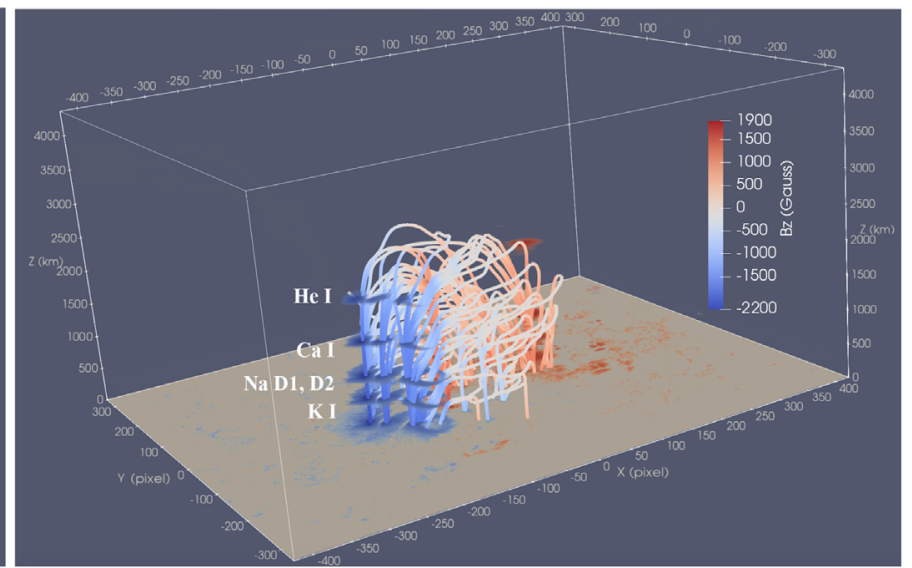

Fig. 4. The figure illustrates the three-dimensional magnetic field of (a) AR AR11166 (left) and (b) 12192 (right). The red-blue colour bar refers to the positive-negative polarity vertical $B_{z}$ magnetic field values measured at the solar surface and computed as function of heights in the solar atmosphere. Particular heights outlining approximately the heights of the line formation of K I, Na D, Ca I, and He I are highlighted.

the lower solar atmosphere, this would then yield the best possible flare onset forecast.

\subsubsection{D magnetic skeleton of active regions}

It has recently been shown by Korsós et al. (2020a) that potential field $(\mathrm{PF})$ extrapolation approaches are sufficient and there is no need for CPU/GPU-hungry and also time-consuming more complex extrapolations. For a demonstration, here, we use the HMI LoS magnetograms as a photospheric lower boundary for constructing the magnetic skeleton of two random examples, say ARs 11166 and 12192, each embedded in a 3D box (see Fig. 4).

The ARs are tracked by using the Yet Another Feature Tracking Algorithm ${ }^{1}$ (YAFTA; Welsch \& Longcope, 2003; DeForest et al., 2007). For the PF extrapolation, we use the method published by Gary (1989). To extrapolate the PF magnetic fields at every moment, we employed the LFFF IDL extrapolation code from http://www.heliodocs.com ${ }^{2}$, which is based on Gary (1989).

Figure 4 illustrates the three-dimensional magnetic field of AR 11166 (left) and AR 12192 (right), respectively, using the PF method described above. At horizontal crosscuts corresponding to the approximate heights of the line formation of K I, Na $\mathrm{D}, \mathrm{Ca} \mathrm{I}$, and He I chromospheric lines the LoS component of the magnetic field could be observed with a solar telescope (e.g., a SAMM) that is equipped with e.g., K I, Na D, Ca I, and He I filters. The horizontal cuts at the formation height of the He $10830 \AA$ A line would be a height of "sunspot tracker" at the top of the chromosphere. In the next section, the $\mathrm{WG}_{\mathrm{M}}$-method will be applied and evaluated to determine the lead time for flare forecast at cross-cut from the photosphere into the deep chromosphere to demonstrate the power of the concept of SAMM-based SAMNet.

\footnotetext{
${ }^{1}$ YAFTA is available from http://solarmuri.ssl.berkeley.edu/? welsch/public/software/YAFTA

${ }^{2} \mathrm{http}: / /$ www.heliodocs.com/php/xdoc_print.php?file=SSW/packages/ spvm/idl/bff/bff.pro
}

\subsubsection{Application of $W G_{M}$ to $3 D$ magnetic skeleton of active regions}

Korsós et al. (2018) identified the so-called optimum heights above the photosphere where the flare precursors of the $\mathrm{WG}_{\mathrm{M}}$ method appear earliest prior to flares of a simulated AR. This finding has led Korsós et al. (2020a) to test and validate the modelling with real solar active regions. They embarked on analysing the variation of the flare onset lead time for 20 flares taking place in 13 ARs. They found that the lead time estimate can be improved by an additional $2-8 \mathrm{~h}$ when compared to the counterpart lead times obtained only from photospheric LoS magnetic data of the same ARs.

Let us put this in the context of yet missing routine chromospheric magnetic field observations where this gap will be closed up by SAMNet. Figure 5 shows the application of the $\mathrm{WG}_{\mathrm{M}}$ method to the 3D magnetic field of ARs 11166 (left panel) and 12192 (right panel), respectively. The evolution of the value of $\mathrm{WG}_{\mathrm{M}}$ and the distance parameter $D_{p m}$ are plotted at particular heights representing the photosphere (upper left pair) and the line formation heights of $\mathrm{K} \mathrm{I}$ (upper right pair), $\mathrm{Na} \mathrm{D}$ (lower left pair) and Ca I (lower right). The $x$-axis is time, measured in days. For a given pair of panels, the upper panel is the temporal variation of $\mathrm{WG}_{\mathrm{M}}$. The pre-flare behavior of $\mathrm{WG}_{\mathrm{M}}$ is fitted by an $n$ th-order polynomial (red line), where the orange dot corresponds to the maximum of $\mathrm{WG}_{\mathrm{M}}$. The bottom panel demonstrates the evolution of $D_{p n}$. The consecutive maximum-minimum-maximum (orange-blue-orange dots) locations of the fitted $n$ th-degree polynomial highlight the full converging-diverging motion uncovered by $D_{p n}$. For details about how the minimum value of $D_{p n}$ is determined, see e.g., Korsós et al. (2019). The vertical green stripe indicates the moment when the flare occurred. Even a simple visual inspection shows that the characteristic pre-flare features vary as a function of height. The heights are chosen to match the approximate formation heights of the three lines (K I, Na D, and $\mathrm{Ca}$ I) as these are popular lines to measure the chromospheric magnetic field.

Next, we demonstrate the role of optimum height, i.e., the height for achieving the longest gain in the lead time for 

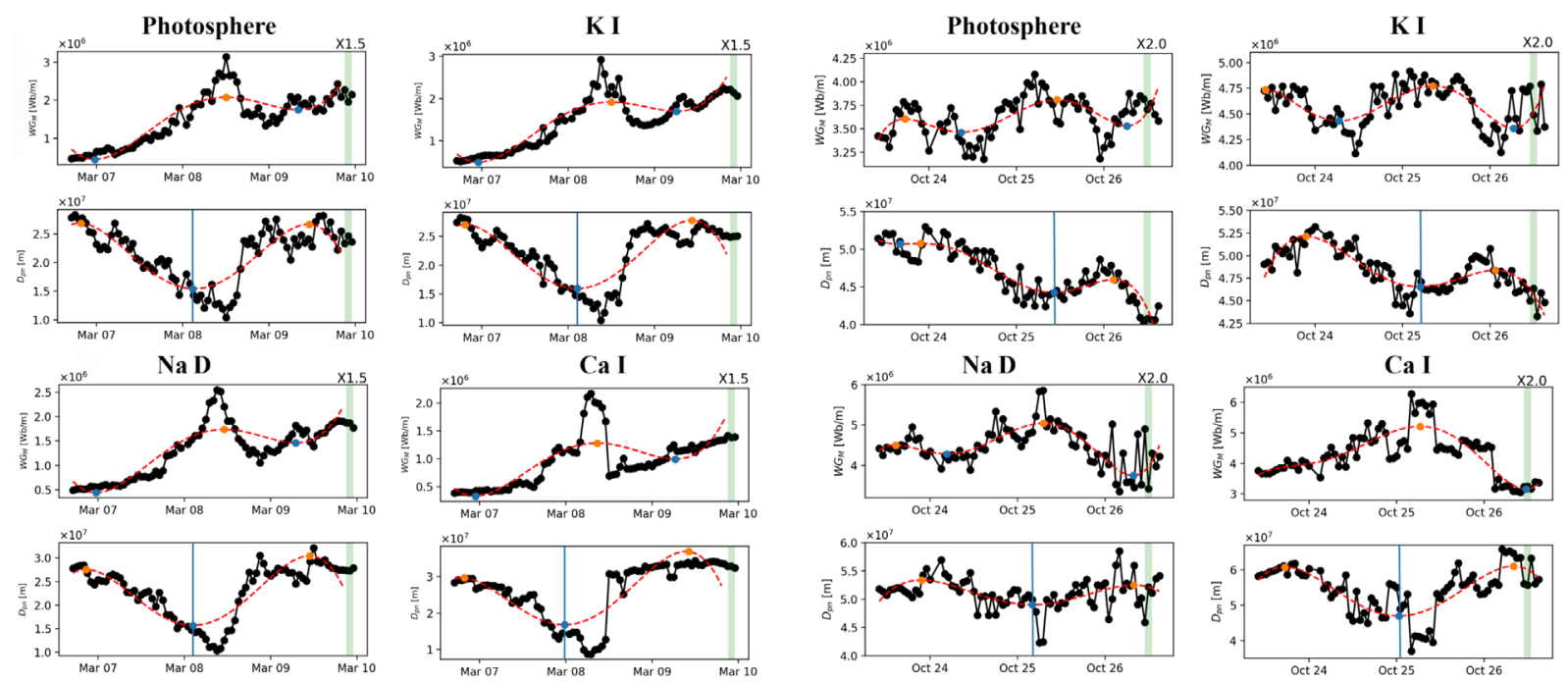

Fig. 5. The figure illustrates the application of the $\mathrm{WG}_{\mathrm{M}}$ method to the $3 \mathrm{D}$ magnetic field of ARs 11166 (left panel) and 12192 (right panel), respectively. The evolution of the value of $\mathrm{WG}_{\mathrm{M}}$ and the distance parameter $D_{p m}$ are plotted at particular heights representing the line formation heights of K I, Na D, and Ca I. The green strip denotes the onset of the X2.0 flare observed. The vertical blue line marks the moment of the closest position of the positive/negative barycentres at a range of heights including the photosphere and the line formation heights of K I, Na D, and $\mathrm{Ca}$ I lines.

estimating the flare onset using the $\mathrm{WG}_{\mathrm{M}}$ method, and whether this height can be found by using a SAMM sentinel equipped with a series of filters (e.g., K I, Na D, and $\mathrm{Ca} \mathrm{I}$, and He I 1083 at a later stage) to measure the required LoS magnetic fields in the lower solar atmosphere.

\subsubsection{Optimum height for forecasting}

The evolution of the $\mathrm{WG}_{\mathrm{M}}$ and the $D_{p n}$ is different at various heights, as has been described above (see Fig. 5). In order to optimise the flare precursor capability of the $\mathrm{WG}_{\mathrm{M}}$ method, let us now identify optimum height(s) in the solar atmosphere. There, the precursor behaviours of the $\mathrm{WG}_{\mathrm{M}}$ and $D_{p n}$ parameters are identifiable earliest prior to each flare. The optimum height(s) is also where these parameters would concurrently yield the earliest signs of pre-flare behavior in time.

First, in Figures $6 \mathrm{a}$ and $6 \mathrm{~b}$ we plot the variation of the start time of the approaching phase (labelled as "Starting"), the moment of the closest approach (labelled as "Minimum"), and the flare onset time of the X1.5 (left panel) and X2.0 (right panel) flares (red line) as a function of height. In Figure 6, the filled grey squares mark the estimated line formation heights for the K I, Na D, Ca I, and He I 1083 magnetically sensitive spectral lines. Most noticeable is that, in general, there are certain heights above the photosphere and well in the chromosphere where the approaching motions began hours earlier and reach the closest point of approach also hours earlier than at the photosphere or at other heights in the solar atmosphere. The actual numerical evaluation shows that the gained lead time is about $3 / 8 \mathrm{~h}$, would a flare prediction have been attempted using chromospheric data, for the X1.5/X2.0 flare, respectively, when compared to their respective photospheric counterparts. This would be a definite (for the X1.5) or an even more considerable (for the X2.0) improvement in determining the flare onset time, would a solar instrumentation measuring routinely LoS magnetic data in the chromosphere. A more comprehensive analysis of Korsós et al. (2020a) has shown that, despite the apparent limitations of employing PF extrapolations only, the potential lead-time improvement for estimating the flare onset time varies in the interval $(2,8)$ hours if one applies the $\mathrm{WG}_{\mathrm{M}}$ method to an identified "sunspot" in the height region between $\sim 800-1000$ and $\sim 1800 \mathrm{~km}$ above the photosphere.

Let us now summarise the main science pillars and findings of Section 2 that support the concept of constructing SAMMs to improve flare forecasting. It is shown that it is sufficient for yielding an efficient and accurate flare forecasting to analyse only the line-of-sight component of the solar magnetic field $B_{\mathrm{LoS}}$, and that analysing $B_{\mathrm{LoS}}$ at a range of heights in the lower solar atmosphere opens up to gain a considerable forecasting lead time. No high resolution is necessary, seeing or diffraction-limited observations would be sufficient. Now, in the following sections, we outline how to realise these science pillars in practice with a facility that will continuously monitor the solar atmospheric magnetic field. This will be done by employing specific magneto-optical filters on solar telescopes that have the task to continuously and routinely monitor the lower solar atmosphere.

\section{The magneto-optical filter (MOF)}

This section is addressing the concept of MOF itself explaining the underlying basic idea and frequency transmission (Sect. 3.1) while also showing some trial images (Sect. 3.2). Given the new realisation context and application of MOF technology to space weather, a brief overview is given in order to better understand the limitations and what future options for development may lay ahead. 
(a)

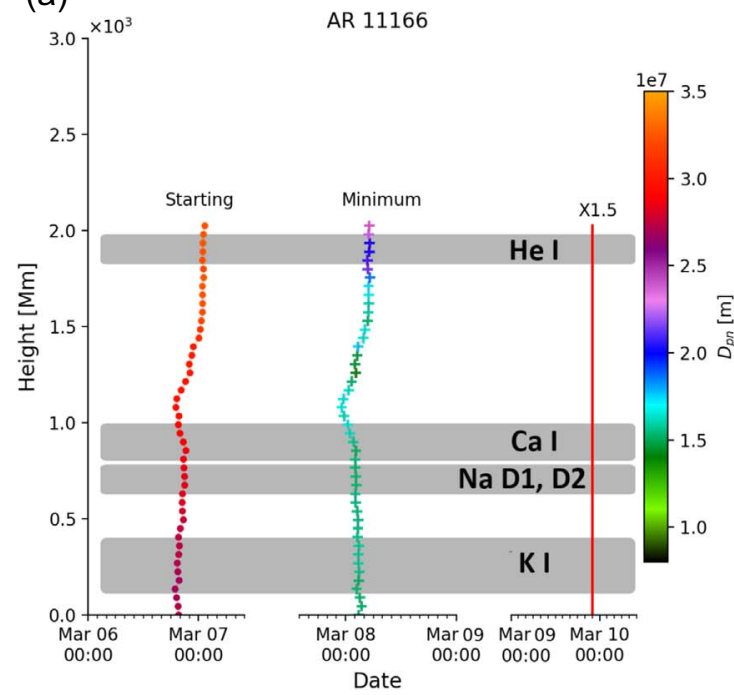

(b)

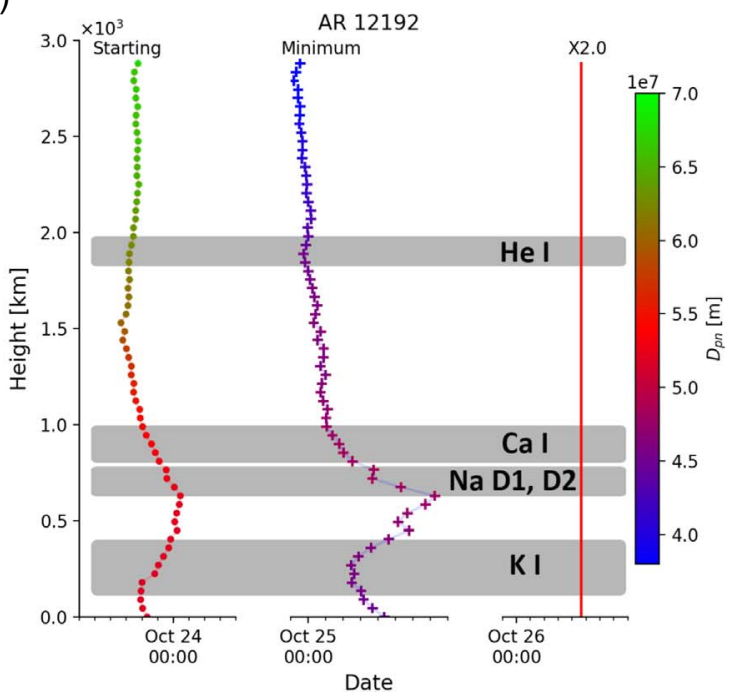

Fig. 6. The figure illustrates the application of the $\mathrm{WG}_{\mathrm{M}}$ method to the LoS component of the 3D magnetic field skeleton of (a) AR 11166 (left) and (b) AR 12192 (right), respectively, to determine optimum forecasting height. The moments of starting and closest convergence (i.e., minimum) times are deduced by the maximum and minimum values, respectively, of the best $n$th degree polynomial fit to the $D_{p n}$ data. The colour bar gives information about the actual value of $D_{p n}$. The grey zones are only indicative line formation heights.

\subsection{The MOF concept}

The magneto-optical filter (MOF) is a frequency-selecting element, which utilises the Faraday effect: the plane of polarisation of linearly polarised light rotates as it propagates through a medium that has an axial magnetic field applied. Linearly polarised light can be thought of as being composed of equal amplitudes of left- (LCP) and right-hand circularly polarised light (RCP). In a circular birefringent medium where the refractive indices for LCP and RCP are different the two components experience different phase shifts, resulting in a net rotation of the linear polarisation. The amount of optical rotation strongly dependent on the dispersion of the medium. When an atomic vapour is used as the dispersive medium, the Zeeman effect splits the atomic absorption lines into two components, $\sigma_{+}$and $\sigma_{-}$ transitions. Hence, the optical rotation only occurs near-atomic resonances, which are intrinsically very narrow. This makes MOFs ideal instruments for filtering out unwanted optical background noise. It is for this reason that the MOF is employed across many disciplines and in solar physics, is referred to as Cacciani Filter after Cimino et al. (1968), Cacciani \& Moretti (1994), Cacciani et al. (1997a, b). In addition to solar physics, magneto-optic filters also find a wide range of applications in other disciplines. Examples include e.g., filtering of frequencydegenerate photon pairs (Zielińska et al., 2014); quantum key distribution (Shan et al., 2006), laser-frequency stabilisation (Miao et al., 2011; Keaveney et al., 2016; Chang et al., 2019); Doppler velocimetry (Bloom et al., 1993); atmospheric LIDAR (Yang et al., 2011; Xia et al., 2020); and simultaneous atmospheric wind and temperature measurement (Huang et al., 2009).

Figure 7 illustrates the principle of using the complete MOF instrument necessary for photospheric observations, which consists of a filter section MOF and a wing selector MOF. Examples of the transmission profiles through a filter section sodium MOF, for one particular input polarisation, are shown in Figure 8. A wavelength of $0 \mathrm{~mA}$ refers to the unperturbed sodium transition $\left(3^{2} \mathrm{~S}_{1 / 2} \rightarrow 3^{2} \mathrm{P}_{3 / 2}\right)$, i.e., atomic resonance. Typically, the spectrum has two wings, in Figure 8b, split from the atomic resonance by the Zeeman effect. The wing bluedetuned from the atomic resonance, i.e., the wing on the left of $0 \mathrm{~mA}$ in Figure 8b, is referred to as the blue wing $(B)$ and the wing on the right of $0 \mathrm{~m} \AA$ is known as the red wing $(R)$.

Depending on the application, the transmission through the filter section MOF can be tailored to give desirable features. For solar chromospheric observations, a transmission that has a single peak centered on the atomic transition is required (Fig. 8a), whereas, for both Doppler- and magnetic-field observations, a transmission that has two wings is necessary (Fig. 8b). The transmission shape and passband can be tailored by choosing appropriate filter section MOF parameters - software has been developed by Yang et al. (2011) to optimize these parameters, which agree with experimental transmission profiles as shown in Figure 8.

For photospheric observations, where a filter section MOF transmission with two wings is necessary, a second MOF is required to extinguish the unwanted wing. This is referred to as the "Wing Selector MOF" in Figure 7. The light that exits the filter section MOF, and subsequently enters the wing selector MOF, can be decomposed into RCP and LCP. For the red wing, the LCP component is absorbed by the wing selector MOF, whereas for the blue wing, the RCP component is absorbed. Using a quarter waveplate and polarising beam splitter (PBS) after the wing selector enables the LCP and RCP components to be separated, such that light in the blue wing is detected on CMOS1 $(B)$ and light in the red wing is detected on CMOS2 $(R)$. Since the input polarisation to the filter section MOF can be set to address either $\sigma_{+}$or $\sigma_{-}$transitions, there are a total of four images that can be taken: $R^{+}$and $B^{+}$ for $\sigma^{+}$input and; $R^{-}$and $B^{-}$for $\sigma_{-}$input. 


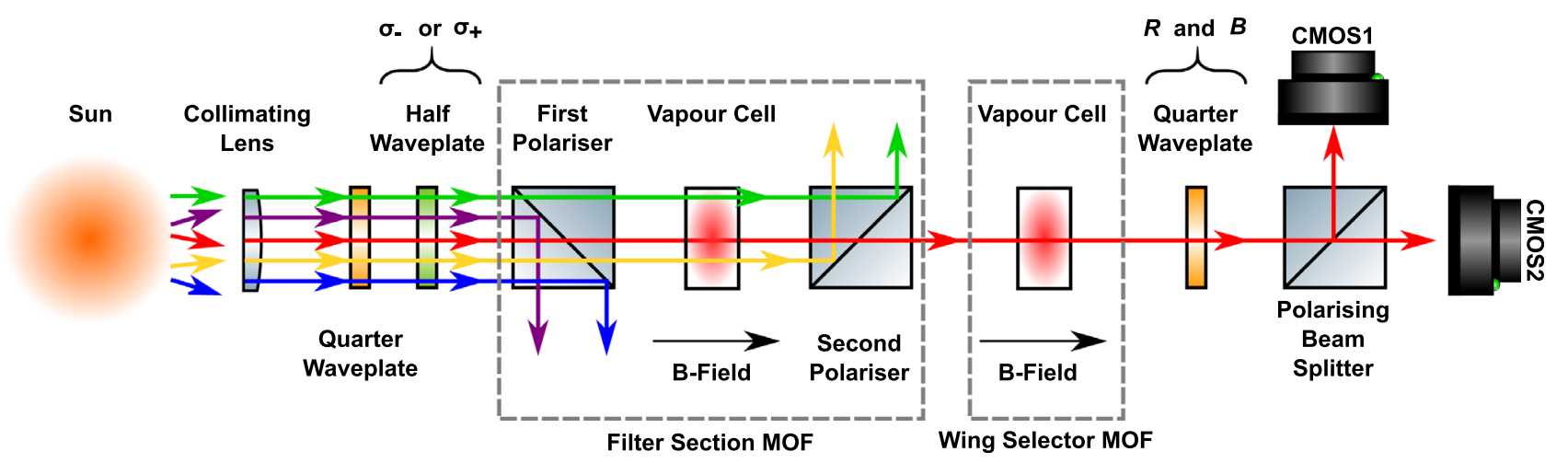

Fig. 7. A schematic illustration of the complete MOF instrument for photospheric observations. Collimated sunlight passes through a two-stage filtering process. The filter section consists of an atomic vapour subject to an axial magnetic field placed between crossed polarisers. The temperature of the vapour and the strength of the magnetic field are chosen to achieve a suitable narrow transmission profile - with two wings centred on the atomic resonance. The second vapour cell and quarter waveplate are used to encode the transmission of the opposite handedness of circularly polarised light into vertical and horizontally polarised light, which is subsequently imaged by cameras CMOS1 and 2, respectively.
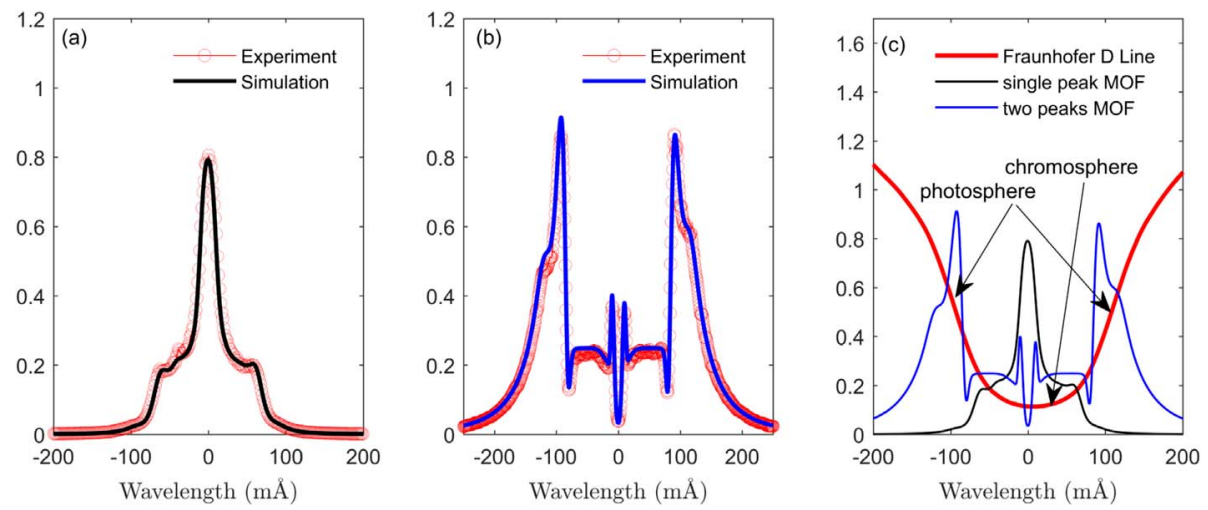

Fig. 8. The simulation and experiment results of the sodium MOF transmission functions. (a) and (b) show the transmission functions of Na D MOF with a center peak and with two wing peaks, respectively. Panel (c) shows the sodium MOF filter that has a single peak in the Fraunhofer D2 line center, enabling chromospheric filtering. The red solid line is the Fraunhofer line and the blue and black solid line are the MOF transmission functions.

Using these four images, the velocity $v_{\text {LoS }}$ and magnetic field $B_{\mathrm{LoS}}$ in line-of-sight can be derived:

$$
\begin{aligned}
B_{\mathrm{LoS}} & \propto \frac{\mathrm{R}^{+}-\mathrm{B}^{+}}{\mathrm{R}^{+}+\mathrm{B}^{+}}-\frac{\mathrm{R}^{-}-\mathrm{B}^{-}}{\mathrm{R}^{-}+\mathrm{B}^{-}}, \quad \text { and } \\
v_{\mathrm{LoS}} & \propto \frac{\mathrm{R}^{+}-\mathrm{B}^{+}}{\mathrm{R}^{+}+\mathrm{B}^{+}}+\frac{\mathrm{R}^{-}-\mathrm{B}^{-}}{\mathrm{R}^{-}+\mathrm{B}^{-}} .
\end{aligned}
$$

Measuring $v_{\mathrm{LoS}}$ and $B_{\mathrm{LoS}}$ serve as strong boundary conditions for modelling solar magnetic fields, particularly in the lower region of the solar atmosphere where the magnetic field is highly non-force-free.

\subsection{Application of MOF to observing the solar atmosphere}

The MOF cell was first envisaged and used for solar applications, in particular in the context of observations of the lower solar atmosphere, by Cimino et al. (1968, 1970), Cacciani et al. (1990, 1997a, b). Full disk maps of the Doppler velocity and magnetic field strength at the photosphere can be obtained through the use of the vapour cells coupled with polarisation active electro-optical components (see, e.g., Oliviero et al., 2002; Finsterle et al., 2004b; Murphy et al., 2005; Moretti et al., 2010; Forte et al., 2017, 2018).

The formation height of the line center of the Fraunhofer $\mathrm{Na}$ $\mathrm{D} 2$ is in the chromosphere and those of the line wings are in the photosphere (Kuridze et al., 2016). The single peak MOF selected the line center and blocked most of the line wings of the Fraunhofer Na D2, therefore the chromosphere could be observed.

A sodium MOF with a single peak and the sodium MOF with two peaks were employed to observe the Sun. Figure 9a shows a test magnetic skeleton of two ARs, namely ARs 11745 (upper left sunspot group) and NOAA AR 11744 (sunspot group in the middle right) image of the lower solar atmosphere. The test observation is to demonstrate the application of a MOF-based approach of observing the lower part of the solar atmosphere with the two peaks MOF. Underneath the MOF image is the corresponding HMI magnetogram. The chromosphere was observed with the single peak MOF as seen in Figure 9b, top view. This example demonstrates how the LoS component of the magnetic field can be obtained and 
(a)

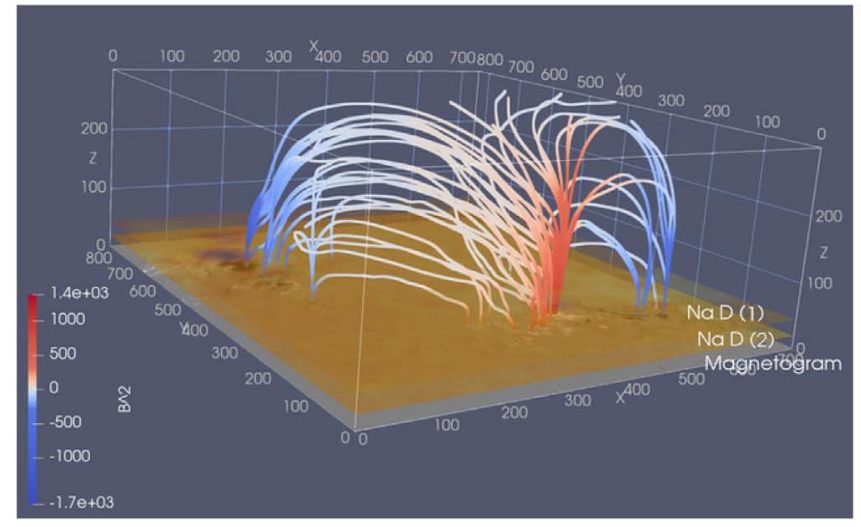

(b)

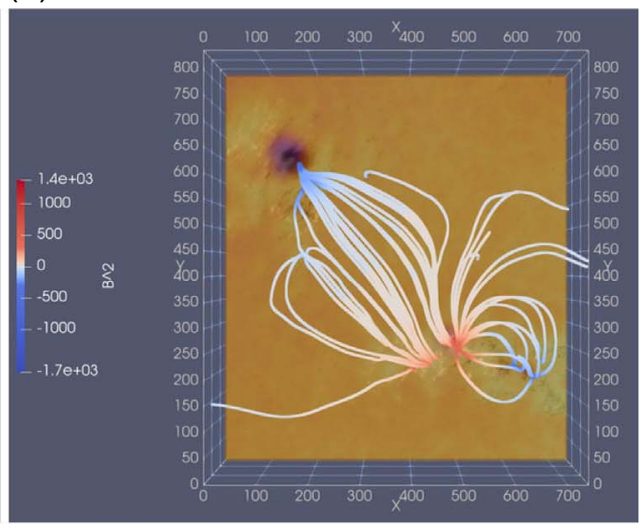

Fig. 9. Observing the lower solar atmosphere. The $x$ - and $y$-axis represent the position in pixels. One pixel corresponds to $0.3 \mathrm{Mm}$. The resolution of the $z$-axis is one-tenth of the resolution of $x$ - and $y$-axis. (a) Left is the visualisation of the lower solar atmosphere embracing NOAA AR 11745 (upper left sunspot group) and NOAA AR 11744 (sunspot group in the middle right). The bottom panel is the photospheric magnetogram, the top panels are taken by the sodium MOF with two peaks $(600-700 \mathrm{~km})$ and a single peak (800-850 km) at UT20130513T030854. Because the vertical scale is much larger than the horizontal one, the bottom panel is hardly visible. (b) Right is the view from the top.

constrained by observations in the most critical (optimum) height of the lower solar atmosphere. Taking such images at a cadence, say 10 mins at fixed wavelengths, would enable to apply the $\mathrm{WG}_{\mathrm{M}}$ (or any other similar) method for flare warning.

Here, we only show an example of the use of the Na D line (Uitenbroek, 2006; Kuridze et al., 2016) with aMOF-based solar telescope. However, other lines (e.g., K I, Ca I, or even He $10830 \AA$ ) with different formation heights (see e.g., for K I Quintero Noda et al., 2017; Ca I Supriya et al., 2014, and for He I Avrett et al., 1994; Leenaarts et al., 2016) would provide $B_{\mathrm{LoS}}$ at further heights, yielding additional constraints on limiting the freedom of constructing the magnetic skeleton in the lower solar atmosphere even with PF method. Once such a skeleton is obtained, given that the PF methods are executable almost instantaneously, even on a modest laptop, approximately near real-time flare warning could be issued. This is the true strength of a MOF-based solar telescope, the core to SAMNet.

\section{The tuneable magneto-optical filter (T-MOF)}

Here, we now briefly discuss the novel idea of the tuneability of a MOF by changing its cell temperatures. When the tuneability capability of MOFs is fully developed, this will have important implications in space weather, solar and astrophysics applications. An initial application to solar physics is shown via a series of intensity images as a result (see Fig. 10) and an associated example of simulations of a sample sodium MOF line width with the demonstrated effect of tuneable filtering (see Fig. 11). The tuneability of MOF is a yet uncharted approach while being a competitive alternative to other, well-established measuring techniques of the solar magnetic field, e.g., by Fabry-Perot or Lyot filters.

During our solar observations at the SAMNet Wuhan Solar Observing Station on 30 July 2013, the variation of temperature of the MOF cell during the observations was tested. The initial temperature of the MOF was varied from about $160{ }^{\circ} \mathrm{C}$ to
$220{ }^{\circ} \mathrm{C}$. At the same time, the passbands of the wing-selector MOF may also need to be tuned (by changing the magnetic field for the wing selector). The associated full disk snapshots are shown in Figure 10. The snapshot in the left top is for the initial, lowest temperature of the MOF, and the snapshot in the bottom right is for the highest one. The temperature and UTC time are labeled for each snapshot. The initial chromospheric features are shown in the top left (Fig. 10a). By varying the temperature, one scans through the line and arrives at observing the solar photospheric features as seen in the snapshot in the bottom right (Fig. 10h). Given the behaviour of the Na D line (see Fig. 11 or Yang et al., 2011), each temperature of the MOF maps a relatively narrow band of the solar atmosphere. We note that with the temperature tuning capability the cadence is slightly varying when compared to observations at a fixed wavelength as time is now needed for the vapour to settle to its newly adjusted temperature. However, on average, a line -and therefore the lower solar atmosphere- can still be scanned in about 30 mins depending on the number of line positions. This is a promising initial result and well suited for further constraining the muchneeded magnetic skeleton of the lower solar atmosphere as even just intensity contours may serve to be a guidance for the computation of the local magnetic field strength.

In summary, the transmission function of a given MOF with a fixed atomic cell could then be "tuned" by changing its temperature (or, for the record, even the magnetic field) and observe different heights of the Sun's atmosphere. Note that this is equally true, though not demonstrated here, to other suitable MOF cells, based on other atomic vapours (e.g., K I, Ca I, and He I).

\section{Realisation of MOF-based telescope for solar physics}

\subsection{Solar Activity Magnetic Monitor - SAMM}

Here, after a short historic account, we briefly outline the current realisation of a MOF-based solar telescope that has been 

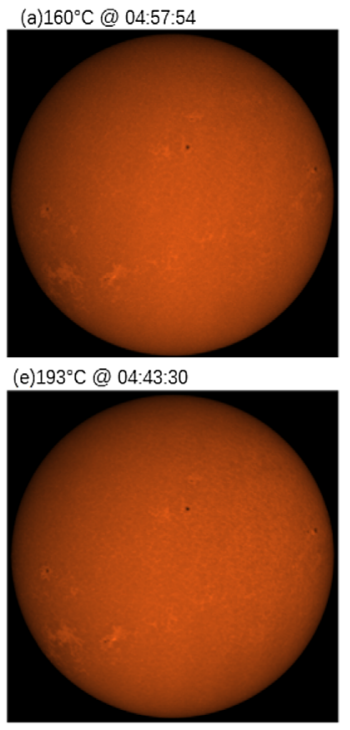

(b) $168^{\circ} \mathrm{C} @ 04: 55.02$

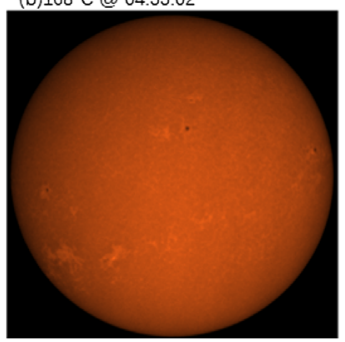

(f)206이 04:40:12

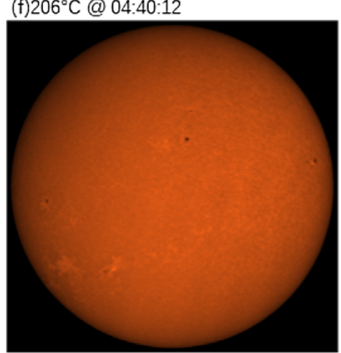

(c) $176^{\circ} \mathrm{C}$ @ 04:50:55

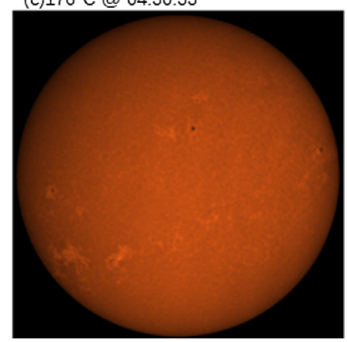

(g) $210^{\circ} \mathrm{C} @ 04: 37 \cdot 56$

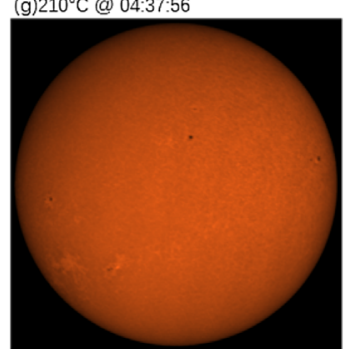

(d) $181^{\circ} \mathrm{C} @ 04: 47 \cdot 14$

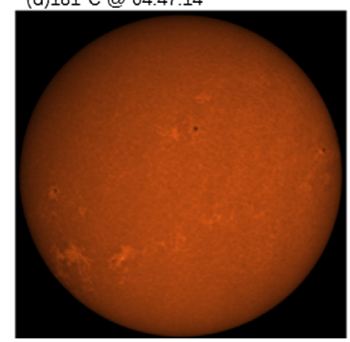

(h) $220^{\circ} \mathrm{C} @ 04: 24: 23$

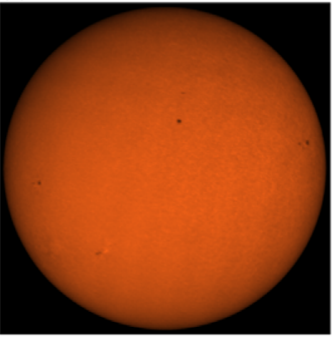

Fig. 10. Observing the lower solar atmosphere by a tuneable sodium MOF application on 30 July 2013, showing the solar intensity. The temperature of the MOF varied from $160{ }^{\circ} \mathrm{C}$ to $220{ }^{\circ} \mathrm{C}$. UTC time and temperature values for each panel are labeled on the snapshots individually. The sequence of images outlines an intensity map scan descending from the mid-chromosphere downward toward the photosphere.
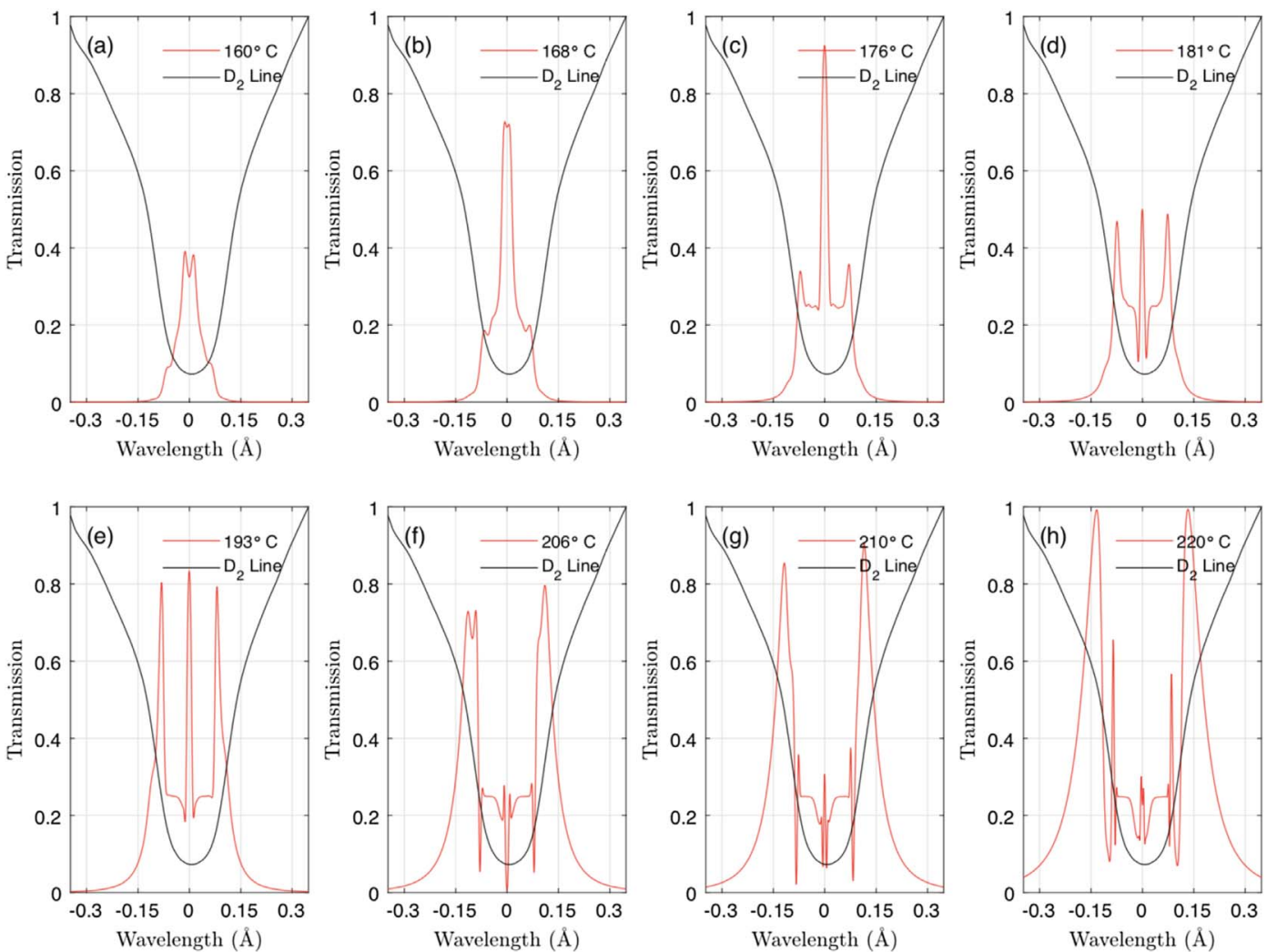

Fig. 11. Simulation results of transmission function for the sodium MOF. The temperature of MOF is changed from $160{ }^{\circ} \mathrm{C}$ to $220{ }^{\circ} \mathrm{C}$. The magnetic field is $200 \mathrm{mT}$, and the length of cell is $25 \mathrm{~mm}$. The $\mathrm{Na}_{2}$ line is from (Wallace et al., 2011).

built on these earlier developments. This new design and development is the so-called Solar Atmospheric Magnetic Monitor (SAMM) station device, that allows to observing routinely the solar $B_{\mathrm{LoS}}$ at various heights (e.g., $\mathrm{K} \mathrm{I}$ and $\mathrm{Na} \mathrm{D}$ ). In this section, we also describe very briefly the optical telescope assemblies (OTAs), cameras, etc. 


\subsubsection{Heritage context}

Agnelli et al. (1975, 1976) introduced preliminary observations by the MOF Na D lines and obtained one of the first images of the Sun by this technology on 11 May 1974 at UT11:40 (see their Fig. 5a). The specific idea of measuring lower solar atmospheric velocities was further explored by Cacciani \& Fofi (1978)] and it was applied to measuring the Doppler signatures of the global solar acoustic $(p)$ modes (e.g., Rhodes et al., 1984; Tomczyk et al., 1993, 1995). In these papers, the practical realisation of the working principles of a MOF-based instrument, earlier only shown in theory by e.g., Cimino et al. (1968, 1970), Beckers (1970), Beckers \& Wagner (1970), and Cacciani et al. (1971), was described in details with the associated transmission profiles. These pioneering studies were among the first to demonstrate how the underlying MOF-based concept can be put for real solar observations.

VAMOS (Velocity And Magnetic Observations of the Sun) - a solar atmospheric MOF imager, based on Cacciani \& Fofi (1978), Moretti et al. (1997), has emerged some decades later. The first version of this instrument (Cacciani et al., 1997a; Moretti et al., 1997) with two sodium vapour cells observed in the Na I D (Oliviero et al., 1998a, b). A newer $40 \mathrm{~cm}$ aperture telescope with two potassium vapour cells became operational in May 1999 located at the Capodimonte Observatory (see Severino et al., 2001 or Fig. 12 in Oliviero et al., 2002).

VAMOS obtained high-cadence observations of the Sun's intensity, velocity, and longitudinal magnetic field component at the photospheric level at the K I $769.9 \mathrm{~nm}$ line formation height. The instrument's main characteristics are listed in Table I of Oliviero et al. (2002).

MOTH-II (Magneto-Optical Filter at Two Heights) - One of the earliest manifestations of developing a MOF-based solar telescope was the MOTH Doppler-magnetograph, upgraded to MOTH-II with two identical instruments, except the actual MOF cells and the narrowband interference filters. The MOTH device is described in Finsterle et al. (2004a), and a good overview can also be found here. The concept of MOTH-II is identical to prototype SAMM's: deliver with a pair of, in this case, $20 \mathrm{~cm}$ aperture OTAs measured full disk Dopplergrams and magnetograms at two heights in the lower solar atmosphere. The K I line is used for photospheric and Na D2 line for lower chromospheric measurements. About the data pipeline and the superb observational results see Forte et al. (in this volume) and here. Excellent applications, e.g., three-dimensional topography of the solar chromospheric canopy (see Finsterle et al., $2004 \mathrm{~b}$ ), the observing of traveling acoustic waves in the lower solar atmosphere (Haberreiter et al., 2007), or even potential space-based applications (Stangalini et al., 2011) all demonstrate the power of acquiring the magnetised lower solar atmosphere by MOF-based solar instrumentation.

$S A M M$ - A technical realisation initially led by Avalon Instruments, supported by an initial start-up incentive from the Ministero per lo Sviluppo Economico (Ministry for Economic Development, MiE), Italy and additional supports from the UK (via STFC, U of Sheffield, and QUB) as well as from Hungary, the very first stage of a further proof-of-concept dual-channel robotic telescope has already been initiated, designed, built and commissioned with 2 OTAs (using K I and Na D MOFs, see for the technical details at Stangalini et al. (2018) and hspf.eu/filters.html). This $2 \times$ OTA solar

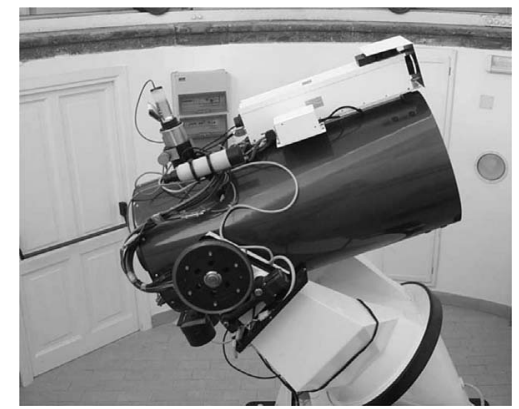

Fig. 12. VAMOS at the Capodimonte Observatory, Italy. Adopted from Oliviero et al. (2002).

telescope is a scaled-down version of the full SAMM concept (see images on the right of Table 1) to demonstrate synoptic data provision only at two heights in the lower regions of the chromosphere (see the top two rows left of Table 1 for the line, wavelength, formation height range and diffraction limit).

The prototype scaled-down SAMM observatory is an enclosure measuring approximately $2 \mathrm{~m}$ on each side. Given its size, there is enabled flexibility of portability making it suitable for easy deployment, although this latter feature is more a bonus as opposed to being an essential requirement. Irrespective of its size, SAMM is provided with a mini-weather station, including rain sensors and a built-in internal camera for secure remote surveillance of operations. In case of loss of power or unfavourable weather conditions, automated closure of the dome will take place within about a minute thanks to a backup battery and UPS that can provide operation for up to 20 mins. SAMM's own computer unit provides control of both the sentinel and the instrumentation as well. The computer system is a standard PC, currently, an Intel Xeon X5560 with dual CPUs at $2.80 \mathrm{GHz}$ for a total of 8 cores, with $24 \mathrm{~GB}$ of RAM installed (see Stangalini et al., 2018). For the sull-scale SAMM the computer system needs to be updated e.g., with Intel NUCs or similar.

A robotic test instrument, when fully operational, is hosted at Gyula Bay Zoltan Solar Observatory (GSO), the scientific and operational HQ of SAMNet, thanks to an open-ended long-term agreement of in-kind support secured from the Local Govt. at Gyula (Hungary). The robotic dome, now capable of hosting the 2-channel scaled-down version of the current version of the SAMM unit master station and associated initial data processing facilities (currently $64 \mathrm{~TB}$ ) is under calibration testing (Fig. 13).

\subsection{Expanding the capabilities of SAMM}

Currently, there are two potential developments to expand the monitoring capabilities of a SAMM station in order to survey the magnetic field and large-scale dynamics in the solar atmosphere. An option is to add another OTAs equipped with Ca I or He 1083 MOF capability for measuring the LoS component of the chromospheric magnetic field about one $\mathrm{Mm}$ (Ca I) and 1.9 Mm (He 1083) above the solar photosphere. The He MOF is rather challenging (see Murphy et al., 2005) but the Ca I MOF for SAMNet is under development (see Sect. 5.2.1). Another option is to mount coronagraphy 
Table 1. Left: Wavelengths and indicative formation heights of the K I, Na $\mathrm{D}_{1}$, Ca I, and He I 1083 chromospheric lines. Right: Snapshot of the scaled-down SAMM prototype telescope at various stages of its design and development.

\begin{tabular}{lccc}
\hline Line & $\begin{array}{c}\text { Wavelength } \\
(\mathrm{nm})\end{array}$ & $\begin{array}{c}\text { Height } \\
(\mathrm{km})\end{array}$ & $\begin{array}{c}\text { Diffraction limit } \\
\text { resolution }(\mathrm{km})\end{array}$ \\
\hline $\mathrm{K} \mathrm{I}$ & 770 & $3-400$ & 0.71 \\
$\mathrm{Na} \mathrm{D} \mathrm{D}_{1}$ & 590 & $6-700$ & 0.54 \\
$\mathrm{Ca} \mathrm{I}$ & 422 & 1000 & 0.39 \\
$\mathrm{He} \mathrm{I}$ & 1083 & 1900 & 1.19
\end{tabular}

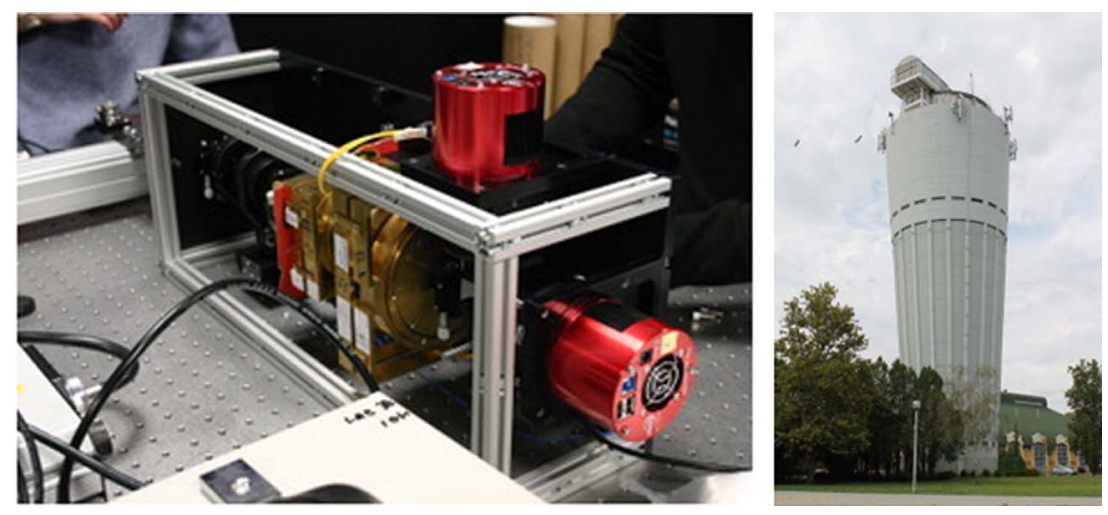

Fig. 13. A new design of thee scaled-down SAMM (left panel) with the refurbished SAMNet HQ, Gyula Bay Zoltán Solar Observatory (GSO) (right panel).

capabilities as a 3rd or 4th OTA, based on the heritage and design of the High-resolution Spectrometer for Coronal Emission Lines (HISCEL) instrument with polarimetry capability. The HISCEL development is led by Aberystwyth University, and up-to-date information is here. This would enable obtaining imaging and velocity information above limb for CMEs lifted off the solar atmosphere.

\subsubsection{The Ca I MOF cell}

Potassium and sodium MOFs are routinely used in solar studies, however, to the best of our knowledge, there is currently the limited application of the Ca-I MOF to solar physics. Although the Ca-I MOF is already commercially available (e.g., at Mojave Solar) and is occasionally used to observe the Sun, there is no routine synoptic observing implementation. The application of a Ca-I MOF to expand the surveying range of the lower solar atmosphere of a SAMM sentinel would be a real advantage because the magnetically-sensitive $\mathrm{Ca}-\mathrm{I}$ line has a formation height that is about one $\mathrm{Mm}$ above the solar surface (Supriya et al., 2014). Observations of $B_{\mathrm{LoS}}$ at these height ranges would further constrain the construction of the $3 \mathrm{D}$ magnetic skeletons of ARs, irrespective of whether there is a small overlap with the formation height of the $\mathrm{Na} \mathrm{D}$ line. Therefore, this would have the additional benefit of improving our capability of delivering additional lead-time gains even further. Moreover, a calcium MOF allows observations of the extremely interesting chromospheric layers of the solar atmosphere where the plasma-beta is approximately one. Ultimately, observing the three different magnetically-sensitive spectral lines (one photospheric and two chromospheric, see Table 1), it is clear that SAMM would produce superior, multi-height synoptic LoS magnetograms.
A calcium filter section MOF (see Fig. 7 for descriptor reference) was simulated utilising the $\mathrm{Ca}\left(4 p^{1} \mathrm{P}_{1}-4 s^{1} \mathrm{~S}_{0}\right)$ strong transition at $422 \mathrm{~nm}$, using an axial magnetic field strength of $B=0.04 \mathrm{~T}$, a cell length of $50 \mathrm{~mm}$, and a vapour temperature of $T=720 \mathrm{~K}$ (Fig. 14). As outlined in Section 3.1, the input polarisation of light, i.e., LCP or RCP, determines whether $\sigma_{+}$or $\sigma_{-}$transitions are addressed, respectively (Adams \& Hughes, 2019). Figure 14a shows the computed absorption coefficients, $\alpha \pm$, for LCP and RCP as a function of detuning (the difference between the frequency of the light and the atomic resonant frequency) in units of the Doppler width, $\Delta v_{D}$. Also shown are the corresponding refractive indices $n_{ \pm}$(Fig. 14b), whereby the differences in the absorption and refractive index curves, concerning LCP and RCP, lead to optical rotation, $\delta(v)$, (Fig. 14b) and filtering effects (Fig. 14d), which are dependent on magnetic field strength. The characteristic two-wing spectral profile is evident in (Fig. 14d), which is necessary for photospheric studies. Further simulations have shown a sensitivity to the vapour temperature and, correspondingly, the number density of the atoms in the cell as well, since this is calculated as a function of temperature (Lide, 1995). Through the use of computational optimisation procedures, we can determine filter parameters that are optimum for our applications of interest (Keaveney et al., 2018).

\section{SAMNet - Solar Activity Monitor Network}

\subsection{The SAMNet concept}

In support of the successful latest development of applying MOF-based technology to solar observation, the Solar Activity Monitor Network was formed of individual identical Solar 

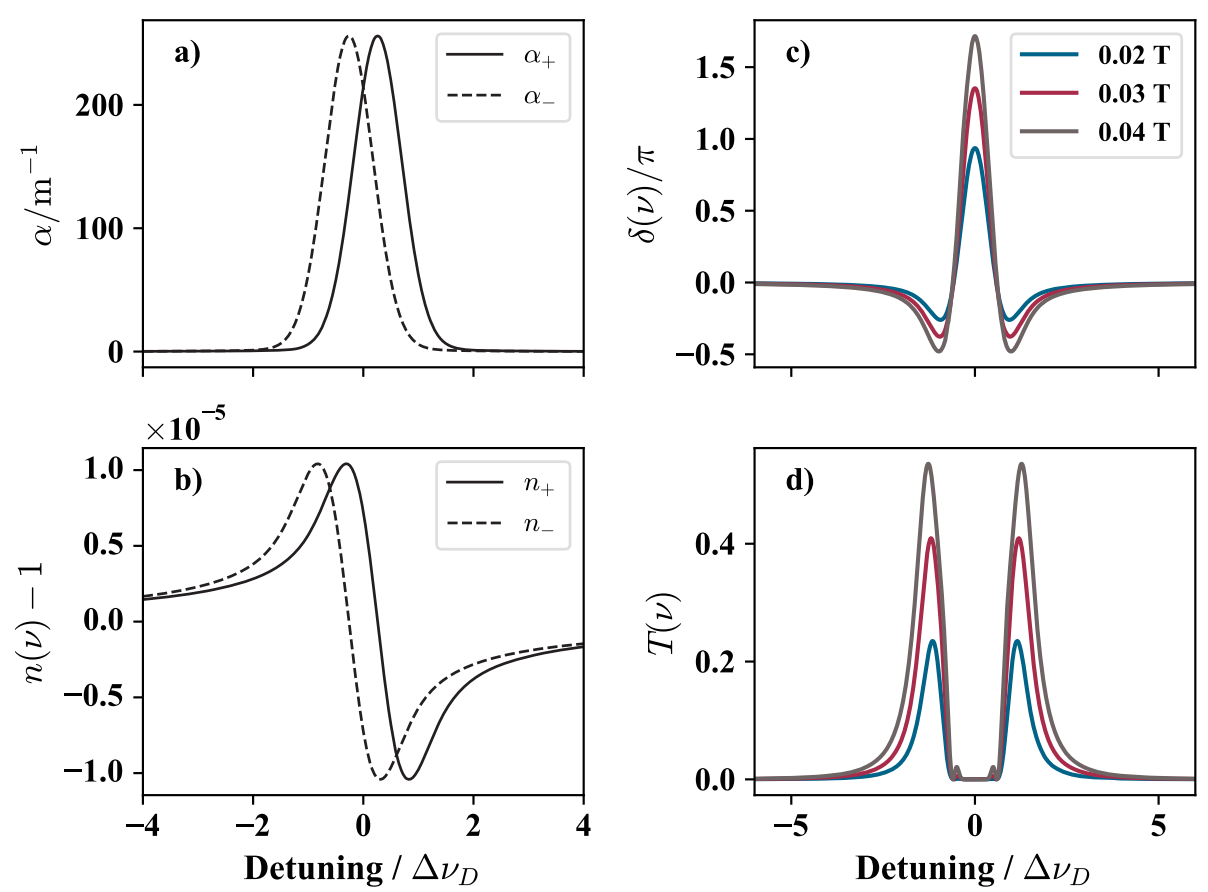

Fig. 14. Graphs showing computed properties of a calcium MOF utilising the $\mathrm{Ca}\left(4 p^{1} \mathrm{P}_{1}-4 s^{1} \mathrm{~S}_{0}\right)$ transition at $422 \mathrm{~nm}$. The abscissa is detuning - difference between the frequency of the light and the atomic resonant frequency - in units of the Doppler width, $\Delta v_{D}$. An applied external magnetic field leads to a splitting of the upper state's energy level through the Zeeman effect. Graph (a) shows the absorption profiles, $\alpha_{+}$and $\alpha_{-}$, for the circular polarisations of the signal light, LHC that drives $\sigma_{+}$transitions, and RHC that drives $\sigma_{-}$transitions, respectively. The magnetic field strength is $0.04 \mathrm{~T}$, and the temperature $T=720 \mathrm{~K}$. In (b) the corresponding dispersion profiles are plotted for the $\sigma_{+}$and $\sigma_{-}$ transitions for a cell of length $50 \mathrm{~mm}$. The Faraday rotation angle, $\delta(v)$, is plotted in (c) for varying magnetic field strength. Note that optical rotations of the order, or greater than, $\pi$ are generated for these parameters, as is necessary for efficient atomic filtering. In (d) the transmission profile is calculated for each of the associated Faraday rotation angles in (c). The characteristic two-wing spectral profile is evident.

Activity Magnetic Monitor (SAMM) solar telescope stations at each partner site when at full capacity. Current international scientific partners with various levels of support and with principal interest include Kanzelhöhe Solar Observatory (Austria); NAOC Beijing, Huairou Solar Observatory, Mingantu Radio Observatory, USTC Hefei, Yunnan Astronomical Observatory, Xinjiang Astronomical Observatory and Innovation Academy for Precision Measurement Science and Technology (China); National U of Colombia, Bogotá (Colombia); Hvar Observatory (Croatia); Abastumani Astrophysical Observatory (Georgia); Eötvös U Budapest and the Hungarian Solar Physics Foundation (Hungary); IIA Bangalore (India); Catania Astrophysical Observatory, Rome Astronomical Observatory (Italy); Pulkovo Observatory, Kislovodsk (Russia); Astronomical Institute of the Slovak Academy of Sciences, Tatranská Lomnica (Slovakia); GONG site at Big Bear Solar Observatory (BBSO), and the Sunspot Solar Observatory of New Mexico State University (USA). The instrumentation suit may be supplied by Dal Sasso - Avalon srl (Rome, Italy) although alternative options are considered too. To achieve maximum performance without spending considerable effort on instrumental cross-calibration across the sentinel stations, it is a highly desired requirement though not necessary- to have identically built SAMM stations at all partner sites. For the geographic distribution of the current SAMNet solar observatory partners see Figure 15. A summary about the geographic location of the partner observatories comprising SAMNet see Table 2. In addition, additional UK expertise (Durham University, QUB, U. of Aberystwyth, UCL/MSSL) with solar technology know-how have also joined the network.

SAMNet is now a UK-led international network with its scientific and observing HQ at Gyula Bay Zoltan Solar Observatory (GSO, see Table 2 for some basic information) under the auspices of HSPF. Once at its peak performance, SAMNet will continuously monitor the Sun's magnetic and velocity fields both in the lower solar atmosphere (from photosphere to upper chromosphere) using a set of identical SAMM sentinel stations equipped with a range of magneto-optical filters (MOFs). Sentinels with suitable observing conditions will also have an HISCEL-based set of coronagraph-mounted spectrograph as well as imaging the low corona using several coronal visible lines to:

- provide advanced warning of adverse space-weather events in the form of solar flares or coronal mass ejections (CMEs) that threaten our high-tech civilisation and the technosphere;

- provide advanced warning of the arrival of CMEs that are already formed and lifted off the solar surface and;

- provide many sought-after continuous synoptic maps (e.g., LoS magnetic and velocity fields, intensity) of the lower solar atmosphere.

The flare and CME onset warning is based on the $\mathrm{WG}_{\mathrm{M}}$ method as outlined in Section 2, while the CME arrival will 


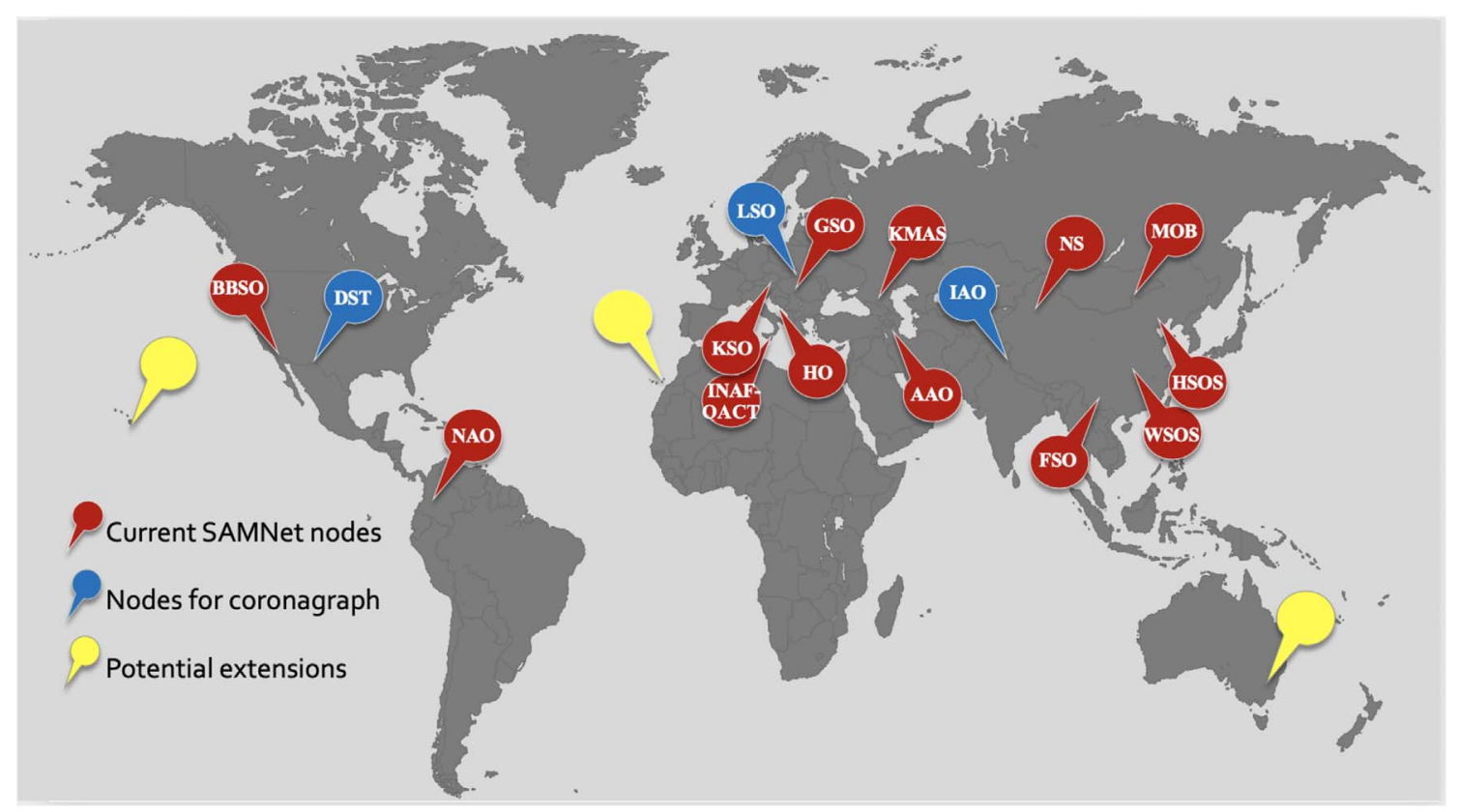

Fig. 15. Potential host sites for future SAMNet sentinels (see Table 2 for further facility details). The blue marks highlight sentinels with about 5-8 h time zone differences to indicate where a coronagraph could be added as 4th OTA. Yellow marks are potential future expansion sites.

Table 2. List of SAMNet partner observatories with their name, abbreviation, longitude, latitude, altitude, and reference to solar instrumentation (if applicable). For FiCoRI see Guevara Gómez et al. (2017), for KSO see Pötzi et al. (2015, 2016, 2018), Veronig \& Pötzi (2016) for the ESA Space Situational Awareness (SSA) programme. For KMAS see here, and for their observational data here

\begin{tabular}{|c|c|c|c|c|c|}
\hline Name & Abbrev & Lat & Long & Alt (m) & Instrument \\
\hline Abastumani Astrophys. Obs. & $\mathrm{AAO}$ & $\mathrm{N} 41^{\circ} 45^{\prime} 15^{\prime \prime}$ & $\mathrm{E} 42^{\circ} 49^{\prime} 10^{\prime \prime}$ & 1650 & $\begin{array}{l}530 \mathrm{~mm} \text { Lyot cor } \\
115 \mathrm{~mm} \text { Lyot cor } \\
\text { dual Ch-Ph telescope }\end{array}$ \\
\hline Fuxian Solar Observatory & FSO & 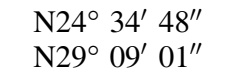 & $\begin{array}{l}\mathrm{E} 102^{\circ} 57^{\prime} 01^{\prime \prime} \\
\mathrm{E} 100^{\circ} 04^{\prime} 13^{\prime \prime}\end{array}$ & $\begin{array}{l}1720 \\
4700\end{array}$ & $\begin{array}{l}\text { NVST Liu et al. (2014) } \\
\text { CGST Deng (2011) }\end{array}$ \\
\hline Huairou Solar Obs. Station & HSOS & $\mathrm{N} 40.32^{\circ}$ & E116.62 ${ }^{\circ}$ & 64 & $\begin{array}{c}35 \mathrm{~cm} \text { SMFT, } 60 \mathrm{~cm} \text { StCT } \\
10 \mathrm{~cm} \text { Fe I vector m.f. } \\
20 \mathrm{~cm} \mathrm{H} \text {-alpha full disk }\end{array}$ \\
\hline Mingantu Observing Base & MOB & $\mathrm{N} 42^{\circ} 12^{\prime}$ & $\mathrm{E} 115^{\circ} 15^{\prime}$ & 1365 & $\begin{array}{c}\text { MUSER Yan et al. }(2009,2021) \\
\text { ISP Yan et al. }(2018)\end{array}$ \\
\hline Nanshan Station & NS & $\mathrm{N} 43.8^{\circ}$ & $\mathrm{E} 87.1^{\circ}$ & 2080 & H-alpha with Lyot filter \\
\hline Wuhan Solar Obs. Station & WSOS & $\mathrm{N} 30^{\circ} 31^{\prime} 54^{\prime \prime}$ & $\mathrm{E} 114^{\circ} 21^{\prime} 26^{\prime \prime}$ & 80 & $\mathrm{Na}$ and $\mathrm{K}$ MOFs \\
\hline Nat. Astron. Obs. Colombia & NAO & $\mathrm{N} 4^{\circ} 38^{\prime} 23^{\prime \prime}$ & $\mathrm{W} 74^{\circ} 05^{\prime} 00^{\prime \prime}$ & 2600 & FiCoRI \\
\hline Gyula Bay Zoltán Sol. Obs. & GSO & $\mathrm{N} 46.649257^{\circ}$ & E21.269346 & 87 & $\begin{array}{l}\text { SAMM master } \\
\text { SAMNet HQ }\end{array}$ \\
\hline Catania Astrophys. Obs. & NAF-OACT & $\mathrm{N} 37^{\circ} 31^{\prime} 43.71^{\prime \prime}$ & $\mathrm{E} 15^{\circ} 04^{\prime} 17.38^{\prime \prime}$ & 197 & $\begin{array}{l}\text { 150-mm Cook ref. } \\
\text { 150-mm H } \alpha \text { Lyot }\end{array}$ \\
\hline Kanzelhöhe Observatory & KSO & $\begin{array}{c}\mathrm{N} 46^{\circ} 40.7^{\prime} \\
\mathrm{N}\end{array}$ & $\begin{array}{c}\mathrm{E} 13^{\circ} 54.1^{\prime} \\
\mathrm{E}\end{array}$ & 1526 & $\begin{array}{l}\text { Global High-Res. } \mathrm{H} \alpha \text { Network } \\
\text { Patrol telescope sys. } \\
\mathrm{H} \alpha \text {, Ca II } \\
\text { Global High-Res. } \mathrm{H} \alpha \text { Network }\end{array}$ \\
\hline Kislovodsk Mountain Astron. Station & KMSA & $\begin{array}{c}\mathrm{N} 43^{\circ} 44.77^{\prime} \\
\mathrm{N}\end{array}$ & $\begin{array}{c}\mathrm{E} 42^{\circ} 31.42^{\prime} \\
\mathrm{E}\end{array}$ & 2070 & $\begin{array}{l}53 \text { and } 20 \mathrm{~cm} \text { coronagraph } \\
\text { STOP telescope }\end{array}$ \\
\hline Lomnicky Stit Observatory & $\mathrm{SO}$ & $\mathrm{N} 49^{\circ} 11^{\prime} 42.53^{\prime \prime}$ & $\mathrm{E} 20^{\circ} 12^{\prime} 47.43^{\prime \prime}$ & 2634 & CoMP-S \\
\hline Sunspot Solar Observatory & SSO & $\mathrm{N} 32.5^{\circ}$ & $\mathrm{W} 105.5^{\circ}$ & 2807 & Dunn Solar Telescope \\
\hline
\end{tabular}


be predicted routinely using a new tool, CME Arrival Time Prediction using Machine Learning Algorithms (CAT-PUMA), developed by Liu et al. (2018). Even if only low corona imaging observations are available, prediction of CME arrival at L1 is still expected as demonstrated by e.g., Wang et al. (2019).

The Consortium of 23 partners from observatories, academia, and industry is committed to investigate the feasibility, design, and when funding is secured to develop its fully-robotic SAMM station system, from dome to fully equipped OTA system. A sentinel may then incorporate (i) magneto-optical filter (MOF) technology and (ii) a coronagraph equipped with a multi-channel spectrograph working in the visible wavelength domain. The technological realisation is associated with the development of the necessary fully automated flare/CME forecast data-processing algorithms. The minimum baseline of a functioning network is a set of SAMM prototypes that consist of a four-strong sentinel station network complemented with the associated fully automated network data processing and control algorithms. Ideally, these four stations would be separated by about $90^{\circ}$ in longitude what would mean a 6-h long time span of active observation per station. Any longer active observation would result in overlap with the added benefit of cross-checking instrument performances.

\subsection{The SAMNet sentinel}

Each SAMNet station will host a sentinel, a fully automated telescope that will monitor the Sun using multiple instruments. These sentinels will be connected to form a global network to provide 24-h solar monitoring. Building on the existing scaled-down SAMM prototype telescope (see Sect. 5.1), here we present an initial design outline for the SAMNet sentinel. Developing a common design simplifies the deployment of new stations in the network, as well as ensuring that all observations will be compatible regardless of which station they originated from.

\subsubsection{Sentinel hardware}

To achieve simultaneous observations of solar active regions at multiple depths, each sentinel will comprise of a single robotic mount with multiple "unit telescopes" (UTs). For sentinels, the components include the various identical elements that support the assembly and electro-mechanical stability, powering of the astronomical equipment, supply of resources, and the basic conditions for the operation and protection of the observatory (power, tower, dome, weather monitoring, mount, tracking, optics, MOF detectors, camera, data acquisition, processing, data transmission, remote control, and automation).

As described in Section 5.1, the existing prototype has two unit telescopes with two SAMM MOF units observing the $\mathrm{K} \mathrm{I}$ and $\mathrm{Na} \mathrm{D}$ lines. The sentinels used by SAMNet will expand on this to hold at least three such unit telescopes, including the existing $\mathrm{K}$ and $\mathrm{Na}$ units alongside the proposed $\mathrm{Ca}$ MOF under development (see Sect. 5.2.1). The unit telescopes will be aligned to point at the same position, and a small guide camera will also be mounted on-axis to ensure the mount reliably tracks the Sun. The potential exists to expand this modular design to include a fourth unit telescope, either holding another MOF unit (e.g., He 1083) or a coronagraphmounted spectrograph.

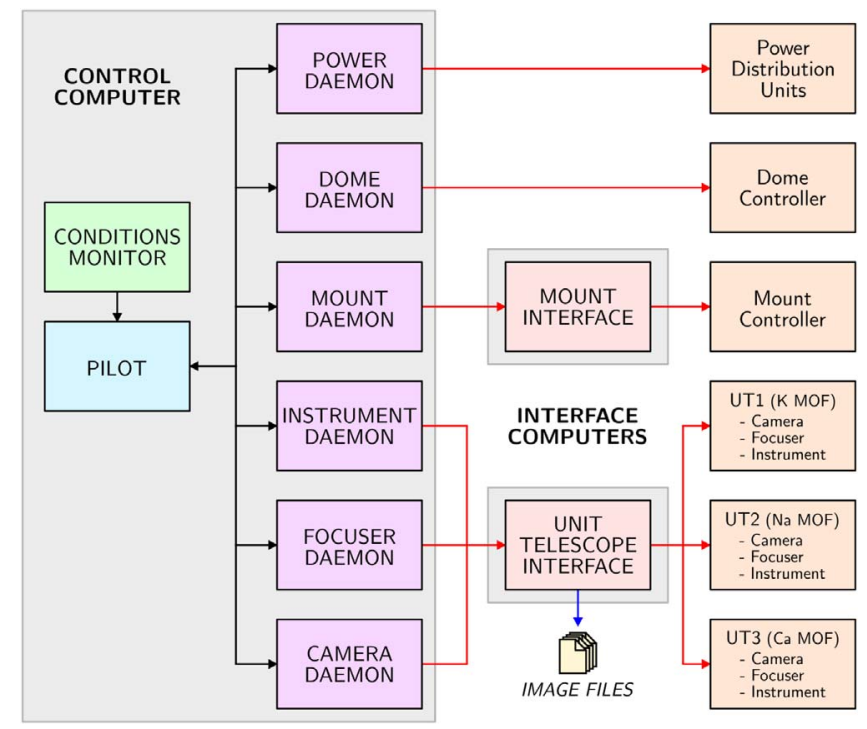

Fig. 16. Proposed control software architecture for a SAMNet sentinel.

It is proposed that the SAMNet sentinels will use similar hardware to their prototype: an array of Celestron OTAs with Baader ND filters, attached to an Astelco mount. The mount may vary subject to the local circumstances at a given station location. By using off-the-shelf hardware as much as possible costs can be reduced and focused on improving the performance of the MOF instruments. The complete SAMNet sentinel node will also include a robotic dome and weather-monitoring stations, to ensure the dome remains closed when the weather is poor.

\subsubsection{Control software}

Each SAMNet sentinel will be fully automated, using custom software that autonomously manages the telescope hardware and daily operations. The proposed system will be based on the control system developed for the Gravitational-wave Optical Transient Observer (GOTO) observatory (Dyer et al., 2018). The GOTO project uses a very similar hardware design to that proposed for SAMNet: each GOTO mount has eight-unit telescopes, and multiple mounts across the world are connected to form a global network. The control software will comprise multiple independent control daemons which are supervised by a master control program known as the "pilot". An initial design for the core software architecture is shown in Figure 16.

The primary elements of the control system will be the hardware daemons. A daemon is computer program that runs as a background process, and each category of hardware has a dedicated control daemon. For example, the dome and mount daemons send commands to the dome and mount respectively, while the camera daemon centralises commands to all cameras across the three-unit telescopes to ensure exposures are synchronised. Each daemon will monitor its associated hardware and then execute commands when received via a command-line script, or, in robotic mode, from the pilot. The pilot is the program in overall control of each sentinel when it is operating in robotic mode. During the day the pilot will ensure observations are 


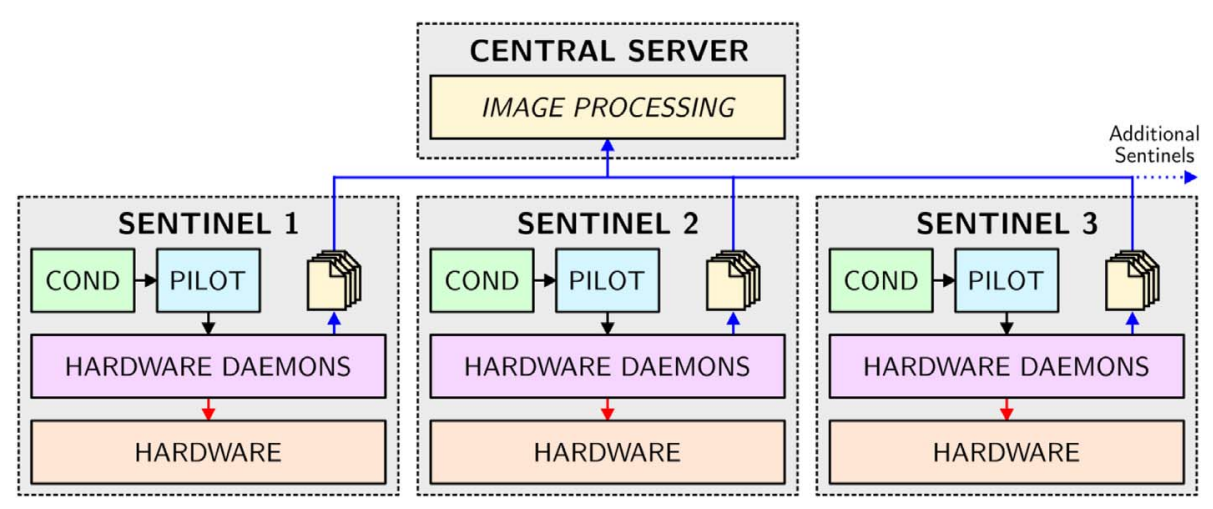

Fig. 17. A schematic design of multiple SAMNet nodes, expanding on Figure 16.

carried out as required, by issuing commands the hardware daemons. The final program will be the conditions monitor, which continuously monitors local weather stations and other system conditions. Should the weather turn bad or other problems occur the conditions monitor will instruct the pilot to pause and close the dome until it is clear to resume observations.

\subsection{Forming a global network}

The central concept of the SAMNet network is to deploy multiple identical sentinels at sites across the Globe. This will make it possible to continuously monitor the Sun for any possible activity, and to issue alerts with as much notice as possible. Each SAMNet site will host one of the sentinels described in Section 6.2, and therefore each sentinel is designed to act as a single node in the wider network. As described in Section 6.1 least four sites spread equally across the Globe are planned for 24-h coverage of the Sun, however, any additional sites will provide redundancy for poor weather or other problems. Figure 15 shows potential host sites proposed by the existing SAMNet partners, each of these sites are listed in Table 2.

Figure 17 shows how each sentinel will transfer images back to the central server for processing. Once the images are transferred, they will be made science-ready and stored at the Sheffield Solar Catalogue (SSC). There, they will be contoured and automatically evaluated by the $\mathrm{WG}_{\mathrm{M}}$ method for flare warning. A warning will be issued with probabilities at $6,12,18$, and $24 \mathrm{~h}$ lead times for a given geographic longitude. The method itself, when applied to photospheric data is capable of forecasting with an about 2-h lead time at very high accuracy (subject to selection criteria). Using the 3D magnetic skeleton data thanks to a SAMM sentinel output at a given longitude, there would be an additional 2-8 $\mathrm{h}$ gain in lead time. However, since there is a deployment of SAMMs around the Globe, the lead time may be further improved at a fixed geographic location by a further up to $16 \mathrm{~h}$. The total aggregated gain in flare warning would therefore be between 20 and $26 \mathrm{~h}$.

\section{Conclusions}

The Sun is the main driver of adverse space weather that causes substantial socioeconomic impact on Earth. This includes both direct effects on specific industry sectors (such as electric power, spacecraft, and aviation industries) and indirect effects on dependent infrastructures (such as positioning and navigation systems, oil/gas pipelines, and financial services). The Lloyd's insurance threat-assessment 2013 report concluded that extreme space weather events would cause USD2.6 trillion damage, and the Space Studies Board, a unit of the National Research Council USA, that full recovery would take 4-10 years (see here). Superstorms aside, however, the "nominal" space weather causes an economic loss of Eur10 billion per annum (Schrijver, 2015).

The network of full 3(+1)-channel SAMM instruments, with SAMNet's cutting-edge MOF and possible coronagraph instrumentation technology, would provide significant improvements to the forecasting of the valiant solar eruptions - the main driver of the extreme space weather events on Earth. This forecast would enable society to take preventive steps to significantly lower the impact of solar eruptions on our technological infrastructure. The mitigation may take place by means based on measuring the LoS components of the solar magnetic and velocity fields in the lower solar atmosphere. In his paper, on page 21, Beckers (1971) says "The magnetic field is however one of them, if not the, most important physical quantity in the observable solar atmosphere. Its measurement by any available method is therefore of the greatest importance". SAMNets with its full-scale SAMM sentinel suit is proposed exactly in this spirit. A single SAMM instrument has a relatively low cost and it requires a modest-size telescope. Potentially, this could allow much broader participation in the SAMNet, especially from the Nations with modest budgets for their national science research including countries in African and South American continents. The instruments could also play a significant role in training the next generation of scientists and the engineers.

SAMNet at its full capacity will yield, for the first time, the foundations of an integrated global space-weather prediction service to mitigate the destructive consequences of space weather. Further, MOF cell spectroscopy has an additional wide range of applications from atomic physics, frequency stabilisation in areas such as laser cooling and trapping, high-precision frequency meteorology, remote temperature sensing, atmospheric LIDAR, Doppler velocimetry, optical communications, and quantum key distribution in free space, quantum optics, 
missile defense to navy, to name a few. Most industrial experiments are carried out using heavier alkali metals, however, there is now a clear rise in applying lighter elements, like Ca.

In the context of other current and future solar observations, SAMM sentinels with their MOF-based sensors and additional coronagraphy instrumentation will also complement data from other space- and ground-based instruments (e.g., the L5 Lagrange mission). In particular, for space-based operational facilities, SAMNet will be an ideal, low-cost easy to maintain facility with no telemetry issues.

Obtaining information about lower solar atmospheric magnetic fields, the likely hotbeds of solar eruptions is often a bottleneck. In particular, there is a clear canopy effect in chromospheric magnetograms, which could be difficult to interpret for modelling input having just LoS observations. With SAMM one will be able to investigate and evaluate how the uncertainties the canopy introduces may affect the accuracy of the forecasting capability.

Acknowledgements. RE is grateful to the Science and Technology Facilities Council (STFC, grant numbers ST/M000826/1 and ST/V005979/1) the UK and the Royal Society for enabling this research. RE also acknowledges the CAS Presidents International Fellowship Initiative, Grant No. 2019VMA052 and the TOP CLLD 7.1.1-16-H-ERFA2019-00207-Magyar Napfizikai Alaptvány ID Nr 1-8-2019/P support.MBK acknowledges STFC grant ST/S000518/1 to Aberystwyth University. MBK also acknowledges the open research program of CAS Key Laboratory of Solar Activity, National Astronomical Observatories, No. KLSA201610. RE and MBK both acknowledge the CAS Key Laboratory of Solar Activity, National Astronomical Observatories Commission for Collaborating Research Program for the support received to carry out part of this work. IGH and DP acknowledge support from EPSRC (grant EP/R002061/1). RB acknowledges the support by the Croatian Science Foundation under project 7549 "Millimeter and submillimeter observations of the solar chromosphere with ALMA". RB, $\mathrm{AH}$, and AMV acknowledge the support from the AustrianCroatian Bilateral Scientific Project "Comparison of ALMA observations with MHD-simulations of coronal waves interacting with coronal holes". AK and PG acknowledge the support from grant VEGA 2/0048/20. AAP acknowledges NASA 80NSSC18K1242 grant and grant No. 19-02-00088 by RFBR. Sunspot Solar Observatory is a partnership between NSF (1945705), the National Solar Observatory, the state of New Mexico, and consortium partners. TZ acknowledges funding from the Austrian Science Fund (FWF, project P30695-N27). FZ acknowledges support by the Università degli Studi di Catania (Piano per la Ricerca Università di Catania 2016-2018 - Linea di intervento 1 "Chance"; Linea di intervento 2 "Dotazione ordinaria"; Fondi di Ateneo 2020-2022, Università di Catania, Linea Open Access), by the Italian MIUR-PRIN grant 2017APKP7T on "Circumterrestrial Environment: Impact of Sun-Earth Interaction”. YH acknowledges funding from NSFC (11790300,11790301). DK has received funding from the Sêr Cymru II scheme, part-funded by the European Regional Development Fund through the Welsh Government. The editor thanks Stuart Jefferies and an anonymous reviewer for their assistance in evaluating this paper.

\section{References}

Adams CS, Hughes IG. 2019. Optics $f 2 f$-from Fourier to Fresnel. Oxford University Press, Oxford, UK.

Agnelli G, Cacciani A, Fofi M. 1975. The magneto-optical filter. I: Premilinary observations in Na D lines. Sol Phys 44(2): 509-518. https://doi.org/10.1007/BF00153229.

Agnelli G, Cacciani A, Fofi M. 1976. Errata: "The magneto-optical filter. I: Preliminary observations in Na D lines" [Sol. Phys., Vol. 44, p. 509-518 (1975)]. Sol Phys 46: 272. https://doi.org/10.1007/ BF00157574.

Anastasiadis A, Papaioannou A, Sandberg I, Georgoulis M, Tziotziou K, Kouloumvakos A, Jiggens P. 2017. Predicting flares and solar energetic particle events: The FORSPEF tool. Sol Phys 292(9): 134. https://doi.org/10.1007/s11207-017-1163-7.

Avrett EH, Fontenla JM, Loeser R. 1994. Formation of the Solar 10830 Angstrom Line. In: Infrared solar physics, Rabin DM, Jefferies JT, Lindsey C (Eds.), Vol. 154, p. 35.

Baranyi T, Győri L, Ludmány A. 2016. On-line tools for solar data compiled at the Debrecen observatory and their extensions with the Greenwich sunspot data. Sol Phys 291: 3081-3102. https://doi. org/10.1007/s11207-016-0930-1.

Barnes G, Leka KD, Schrijver CJ, Colak T, Qahwaji R, et al. 2016. A comparison of flare forecasting methods. I. Results from the allclear workshop. Astrophys J 829: 89. https://doi.org/10.3847/ 0004-637X/829/2/89.

Beckers JM. 1970. Narrow band filters based on magnetooptical effects. Appl Opt 9(3): 595. https://doi.org/10.1364/AO.9. 000595.

Beckers JM. 1971. The measurement of solar magnetic fields. In: Solar magnetic fields, Howard R (Ed.). Vol. 43 of IAU Symposium, p. 3.

Beckers JM, Wagner WJ. 1970. A photographic polarimeter for solar observations. Appl Opt 9: 1933-1934. https://doi.org/10.1364/ AO.9.001933.

Bloom SH, Searcy PA, Choi K, Kremer R, Korevaar E. 1993. Helicopter plume detection by using an ultranarrow-band noncoherent laser Doppler velocimeter. Opt Lett 18(3): 244-246. https://doi.org/10.1364/OL.18.000244.

Bobra MG, Couvidat S. 2015. Solar flare prediction using SDO/HMI vector magnetic field data with a machine-learning algorithm. Astrophys J 798(2): 135. https://doi.org/10.1088/0004-637X/798/ 2/135.

Cacciani A, Fofi M. 1978. The magneto-optical filter. II. Velocity field measurements. Sol Phys 59(1): 179-189. https://doi.org/ 10.1007/BF00154941.

Cacciani A, Cimino M, Fofi M. 1971. A short report on the Magnetic Beam Absorption Filter Research at the Rome astronomical observatory. In: Solar magnetic fields, Howard R (Eds.), Vol. 43, p. 94.

Cacciani A, Moretti P-F. 1994. Magneto-optical filter: concept and applications in astronomy. In: Proc. SPIE, Crawford DL, Craine ER (Eds.), Vol. 2198 of Society of Photo-Optical Instrumentation Engineers (SPIE) Conference Series, pp. 219-228. https://doi.org/ 10.1117/12.176751

Cacciani A, Ricci D, Rosati P, Rhodes EJ, Smith E, Tomczyk S, Ulrich RK. 1990. Solar magnetic fields measurements with a magneto-optical filter. Nuovo Cimento C Geophys Space Phys C 13: 125-130. https://doi.org/10.1007/BF02515781.

Cacciani A, Marmolino C, Moretti PF, Oliviero M, Severino G, Smaldone LA. 1997a. Simultaneous Doppler and magnetic solar maps from a MOF installed at the Osservatorio di Capodimonte. Mem Soc Astron Italiana 68: 467. 
Cacciani A, Moretti PF, Rodgers WE. 1997b. Measuring Doppler and Magnetic Fields Simultaneously. Sol Phys 174: 115-128. https://doi.org/10.1023/A:1004935524038.

Campi C, Benvenuto F, Massone AM, Bloomfield DS, Georgoulis MK, Piana M. 2019. Feature ranking of active region source properties in solar flare forecasting and the uncompromised stochasticity of flare occurrence. Astrophys $J$ 883(2): 150. https://doi.org/10.3847/1538-4357/ab3c26.

Camporeale E. 2019. The challenge of machine learning in space weather: Nowcasting and forecasting. Space Weather 17(8): 11661207. https://doi.org/10.1029/2018SW002061, https://agupubs. onlinelibrary.wiley.com/doi/abs/10.1029/2018SW002061.

Chang P, Chen Y, Shang H, Guan X, Guo H, Chen J, Luo B. 2019. A Faraday laser operating on Cs $852 \mathrm{~nm}$ transition. Appl Phys BLasers Opt 125(12): Article ID 230. https://doi.org/10.1007/ s00340-019-7342-5.

Cimino M, Cacciani A, Sopranzi N. 1968. An instrument to measure solar magnetic fields by an atomic-beam method. Sol Phys 3(4): 618-622. https://doi.org/10.1007/BF00151943.

Cimino M, Cacciani A, Fofi M. 1970. Some developments of the magnetic beam absorption filter. Sol Phys 11(2): 319-333. https://doi.org/10.1007/BF00155231.

Cui Y, Li R, Zhang L, He Y, Wang H. 2006. Correlation between solar flare productivity and photospheric magnetic field properties. 1. Maximum horizontal gradient, length of neutral line, number of singular points. Sol Phys 237: 45-59. https://doi.org/10.1007/ s11207-006-0077-6.

DeForest CE, Hagenaar HJ, Lamb DA, Parnell CE, Welsch BT. 2007. Solar magnetic tracking. I. Software comparison and recommended practices. Astrophys J 666: 576-587. https://doi. org/10.1086/518994.

Deng YY. 2011. Introduction to the Chinese Giant Solar Telescope. In: Astronomical Society of India Conference Series, Vol. 2 of Astronomical Society of India Conference Series, pp. 31-36.

Dyer MJ, Dhillon VS, Littlefair S, Steeghs D, Ulaczyk K, Chote P, Galloway D, Rol E. 2018. A telescope control and scheduling system for the Gravitational-wave Optical Transient Observer (GOTO). In: Proceedings of the SPIE, Vol. 10704 of Society of Photo-Optical Instrumentation Engineers (SPIE) Conference Series, p. 107040C. https://doi.org/10.1117/12.2311865.

Eastwood JP, Biffis E, Hapgood MA, Green L, Bisi MM, Bentley RD, Wicks R, McKinnell LA, Gibbs M, Burnett C. 2017. The economic impact of space weather: Where do we stand? Risk Anal 37(2): 206-218. https://doi.org/10.1111/risa.12765.

Finsterle W, Jefferies SM, Cacciani A, Rapex P, McIntosh SW. 2004a. Helioseismic mapping of the magnetic canopy in the solar chromosphere. Astrophys J Lett 613(2): L185-L188. https://doi. org/10.1086/424996.

Finsterle W, Jefferies SM, Cacciani AR, Rapex P, Giebink C, Knox A, Dimartino V. 2004b. Seismology of the solar atmosphere. Sol Phys 220(2): 317-331. https://doi.org/10.1023/B:SOLA.0000031397. $73790.7 \mathrm{~b}$.

Florios K, Kontogiannis I, Park S-H, Guerra JA, Benvenuto F, Bloomfield DS, Georgoulis MK. 2018. Forecasting solar flares using magnetogram-based predictors and machine learning. Sol Phys 293(2): 28. https://doi.org/10.1007/s11207-018-1250-4.

Forte R, Jefferies S, Pietropaolo E, Scardigli S, Giovannelli L, Del Moro D, Berrilli F. 2017. MOTH II calibration pipeline and data merging with SDO/HMI and SDO/AIA. In: SOLARNET IV: The physics of the sun from the interior to the outer atmosphere, p. 122.

Forte R, Jefferies SM, Berrilli F, Del Moro D, Fleck B, Giovannelli L, Murphy N, Pietropaolo E, Rodgers W. 2018. The MOTH II Doppler-magnetographs and data calibration pipeline. In: Space weather of the heliosphere: Processes and forecasts, Vol. 335 of IAU Symposium, Foullon C, Malandraki OE (Eds.), pp. 335-339. https://doi.org/10.1017/S1743921318000029.

Gary GA. 1989. Linear force-free magnetic fields for solar extrapolation and interpretation. Astrophys J Suppl Ser 69: 323-348. https://doi.org/10.1086/191316.

Georgoulis MK. 2013. Toward an efficient prediction of solar flares: Which parameters, and how? Entropy 15(11): 5022-5052. https://doi.org/10.3390/e15115022.

Guevara Gómez JC, Martínez Oliveros JC, Calvo-Mozo B. 2017. First Colombian solar radio interferometer: Current stage. In: Fine structure and dynamics of the solar atmosphere, Vargas Domínguez S, Kosovichev AG, Antolin P, Harra L (Eds.), pp. 16-19. https://doi.org/10.1017/S1743921317000084.

Haberreiter M, Finsterle W, Jefferies SM. 2007. On the observation of traveling acoustic waves in the solar atmosphere using a magneto-optical filter. Astronomische Nachrichten 328(3): 211. https://doi.org/10.1002/asna.200610721.

Hasan SS, Soltau D, Kärcher H, Süß M, Berkefeld T. 2010. NLST: India's National Large Solar Telescope. Astron Nachr 331(6): 628. https://doi.org/10.1002/asna.201011389.

Huang W, Chu X, Wiig J, Tan B, Yamashita C, et al. 2009. Field demonstration of simultaneous wind and temperature measurements from 5 to $50 \mathrm{~km}$ with a Na double-edge magneto-optic filter in a multi-frequency Doppler lidar. Opt Lett 34(10): 1552-1554. https://doi.org/10.1364/OL.34.001552.

Huang X, Wang H, Xu L, Liu J, Li R, Dai X. 2018. Deep learning based solar flare forecasting model. I. Results for line-of-sight magnetograms. Astrophys J 856(1): 7. https://doi.org/10.3847/ 1538-4357/aaae00.

Keaveney J, Hamlyn WJ, Adams CS, Hughes IG. 2016. A singlemode external cavity diode laser using an intra-cavity atomic Faraday filter with short-term linewidth $<400 \mathrm{kHz}$ and long-term stability of <1 MHz. Rev Sci Instrum 87(9): 095111. https://doi. org/10.1063/1.4963230.

Keaveney J, Wrathmall SA, Adams CS, Hughes IG. 2018. Optimized ultra-narrow atomic bandpass filters via magneto-optic rotation in an unconstrained geometry. Opt Lett 43(17): 4272. https://doi.org/ 10.1364/OL.43.004272.

Kim T, Park E, Lee H, Moon Y-J, Bae S-H, et al. 2019. Solar farside magnetograms from deep learning analysis of STEREO/EUVI data. Nat Astron 3: 397-400. https://doi.org/10.1038/s41550-0190711-5.

Korsós MB, Ludmány A, Erdélyi R, Baranyi T. 2015. On flare predictability based on sunspot group evolution. Astrophys $J$ Lett 802(2): L21. https://doi.org/10.1088/2041-8205/802/2/L21.

Korsós MB, Chatterjee P, Erdélyi R. 2018. Applying the weighted horizontal magnetic gradient method to a simulated flaring active region. Astrophys J 857: 103. https://doi.org/10.3847/1538-4357/ aab891.

Korsós MB, Yang S, Erdélyi R. 2019. Investigation of pre-flare dynamics using the weighted horizontal magnetic gradient method: From small to major flare classes. J Space Weather Space Clim 9: A6. https://doi.org/10.1051/swsc/2019002.

Korsós MB, Georgoulis MK, Gyenge N, Bisoi SK, Yu S, Poedts S, Nelson CJ, Liu J, Yan Y, Erdélyi R. 2020a. Solar flare prediction using magnetic field diagnostics above the photosphere. Astrophys J 896(2): 119. https://doi.org/10.3847/1538-4357/ab8fa2.

Korsós MB, Romano P, Morgan H, Ye Y, Erdélyi R, Zuccarello F. 2020b. Differences in periodic magnetic helicity injection behavior between flaring and non-flaring active regions: Case study. Astrophys J Lett 897(2): L23. https://doi.org/10.3847/2041-8213/ ab9d7a. 
Kuridze D, Mathioudakis M, Christian DJ, Kowalski AF, Jess DB, Grant SDT, Kawate T, Simões PJA, Allred JC, Keenan FP. 2016. Observations and simulations of the Na I D1 line profiles in an M-class solar flare. Astrophys J 832(2): 147. https://doi.org/ 10.3847/0004-637X/832/2/147.

Leenaarts J, Golding T, Carlsson M, Libbrecht T, Joshi J. 2016. The cause of spatial structure in solar He I $1083 \mathrm{~nm}$ multiplet images. A\&A 594: A104. https://doi.org/10.1051/0004-6361/201628490.

Leka KD, Barnes G, Wagner E. 2018. The NWRA classification Infrastructure: Description and extension to the discriminant analysis flare forecasting system (DAFFS). J Space Weather Space Clim 8: A25. https://doi.org/10.1051/swsc/2018004.

Leka KD, Park S-H, Kusano K, Andries J, Barnes G, et al. 2019a. A comparison of flare forecasting methods. II. Benchmarks, metrics, and performance results for operational solar flare forecasting systems. Astrophys J Suppl Ser 243(2): 36. https://doi.org/10. 3847/1538-4365/ab2e12.

Leka KD, Park S-H, Kusano K, Andries J, Barnes G, et al. 2019b. A comparison of flare forecasting methods III systematic behaviors of operational solar flare forecasting systems. Astrophys $J$ 881(2): 101. https://doi.org/10.3847/1538-4357/ab2e11.

Lide DR. 1995. CRC handbook of chemistry and physics: A readyreference book of chemical and physical data. CRC Press, Boca Raton, FL.

Liu Z, Xu J, Gu B-Z, Wang S, You J-Q, et al. 2014. New vacuum solar telescope and observations with high resolution. Res Astron Astrophys 14(6): 705-718. https://doi.org/10.1088/1674-4527/14/ $6 / 009$.

Liu J, Ye Y, Shen C, Wang Y, Erdélyi R. 2018. A new tool for CME arrival time prediction using machine learning algorithms: CATPUMA. Astrophys J 855(2): 109. https://doi.org/10.3847/15384357/aaae69.

Liu J, Wang Y, Huang X, Korsós MB, Jiang Y, Wang Y, Erdélyi R. 2021. Reliability of AI-generated magnetograms from only EUV images. Nat Astron 5: 108-110. https://doi.org/10.1038/s41550021-01310-6.

Miao X, Yin L, Zhuang W, Luo B, Dang A, Chen J, Guo H. 2011. Note: Demonstration of an external-cavity diode laser system immune to current and temperature fluctuations. Rev Sci Instrum 82(8): 086106. https://doi.org/10.1063/1.3624696.

Moretti PF, Severino G, Cauzzi G, Reardon K, Straus T, Cacciani A, Marmolino C, Oliviero M, Smaldone LA. 1997. The magnetooptical filter in Napoli: Perspectives and test observations. In: Vol. 225 of Astrophysics and Space Science Library. https://doi.org/ 10.1007/978-94-011-5167-2-32.

Moretti PF, Berrilli F, Bigazzi A, Jefferies SM, Murphy N, Roselli L, di Mauro MP. 2010. Future instrumentation for solar physics: A double channel MOF imager on board ASI Space Mission ADAHELI. Astrophys Space Sci 328(1-2): 313-318. https://doi. org/10.1007/s10509-009-0251-z.

Murphy N, Smith E, Rodgers W, Jefferies S. 2005. Chromospheric observations in the helium $1083 \mathrm{~nm}$ line - A new instrument. In: Solar Wind 11/SOHO 16, Connecting Sun and Heliosphere, Fleck B, Zurbuchen TH, Lacoste H (Eds.), Vol. 592 of ESA Special Publication, p. 511.

Nishizuka N, Sugiura K, Kubo Y, Den M, Ishii M. 2018. Deep flare net (DeFN) model for solar flare prediction. Astrophys $J \mathbf{8 5 8}(\mathbf{2})$ : 113. https://doi.org/10.3847/1538-4357/aab9a7.

Oliviero M, Dolci M, Severino G, Straus T, Cacciani A, Moretti PF. 1998a. VAMOS: Velocity and magnetic observations of the Sun. In: New eyes to see inside the Sun and Stars, Deubner F-L, Christensen-Dalsgaard J, Kurtz D (Eds.), Vol. 185 of IAU Symposium, p. 53.
Oliviero M, Severino G, Straus T. 1998b. The VAMOS data analysis pipeline. In: Structure and dynamics of the interior of the Sun and Sun-like Stars. Korzennik S (Ed.), Vol. 418 of ESA Special Publication, p. 275.

Oliviero M, Moretti PF, Severino G, Straus T, Magrì M, Tripicchio A. 2002. Preliminary results on the solar photospheric dynamics observed with Vamos. Sol Phys 209(1): 21-35. https://doi.org/ 10.1023/A:1020904204842.

Park E, Moon Y-J, Shin S, Yi K, Lim D, Lee H, Shin G. 2018. Application of the deep convolutional neural network to the forecast of solar flare occurrence using full-disk solar magnetograms. Astrophys J 869(2): 91. https://doi.org/10.3847/1538-4357/aaed40.

Pevtsov AA. 2017. Space weather forecasting and supporting research in the USA. Geomagn Aeron 57(7): 769-775. https://doi.org/10.1134/S0016793217070179.

Pötzi W, Veronig AM, Riegler G, Amerstorfer U, Pock T, Temmer M, Polanec W, Baumgartner DJ. 2015. Real-time flare detection in ground-based $\mathrm{H} \alpha$ imaging at Kanzelhöhe observatory. Sol Phys 290(3): 951-977. https://doi.org/10.1007/s11207-014-0640-5.

Pötzi W, Veronig AM, Temmer M, Baumgartner DJ, Freislich H, Strutzmann H. 2016. 70 years of sunspot observations at the Kanzelhöhe observatory: Systematic study of parameters affecting the derivation of the relative sunspot number. Sol Phys 291(9-10): 3103-3122. https://doi.org/10.1007/s11207-016-0857-6.

Pötzi W, Veronig AM, Temmer M. 2018. An event-based verification scheme for the real-time flare detection system at Kanzelhöhe observatory. Sol Phys 293(6): 94. https://doi.org/10.1007/s11207018-1312-7.

Qahwaji R, Colak T. 2007. Automatic short-term solar flare prediction using machine learning and sunspot associations. Sol Phys 241(1): 195-211. https://doi.org/10.1007/s11207-006-0272-5.

Quintero Noda C, Uitenbroek H, Katsukawa Y, Shimizu T, Oba T, et al. 2017. Solar polarimetry through the K I lines at $770 \mathrm{~nm}$. Mon Not $R$ Astron Soc 470(2): 1453-1461. https://doi.org/10.1093/ mnras/stx 1344 .

Ravindra B, Kesavan P, Thulasidharen KC, Rajalingam M, Sagayanathan K, et al. 2018. Installation of solar chromospheric telescope at the Indian Astronomical Observatory, Merak. J Astrophys Astron 39(5): 60. https://doi.org/10.1007/s12036-018-9554-1.

Rhodes EJ, Cacciani A, Tomczyk S, Ulrich RK, Blamont J, Howard RF, Dumont P, Smith EJ. 1984. A compact dopplergraph/magnetograph suitable for space-based measurements of solar oscillations and magnetic fields. Adv Space Res 4(8): 103-112. https://doi.org/10.1016/0273-1177(84)90371-5.

Schrijver CJ. 2007. A characteristic magnetic field pattern associated with all major solar flares and its use in flare forecasting. Astrophys J Lett 655: L117-L120. https://doi.org/10.1086/511857.

Schrijver CJ. 2015. Socio-economic hazards and impacts of space weather: The important range between mild and extreme. Space Weather 13(9): 524-528. https://doi.org/10.1002/2015SW001252.

Severino G, Moretti PF, Oliviero M, Vamos Team. 2001. The velocity and magnetic observations of the Sun (VAMOS) project: Status and future prospects. In: SOHO 10/GONG 2000 Workshop: Helio- and Asteroseismology at the Dawn of the Millennium, Wilson A, Pallé PL (Eds.), Vol. 464 of ESA Special Publication, pp. 337-340.

Shan X, Sun X, Luo J, Tan Z, Zhan M. 2006. Free-space quantum key distribution with $\mathrm{Rb}$ vapor filters. Appl Phys Lett 89(19): 191,121. https://doi.org/10.1063/1.2387867.

Stangalini M, Moretti PF, Berrilli F, Del Moro D, Jefferies SM, Severino G, Oliviero M. 2011. DIMMI-2h a MOF-based instrument for Solar Satellite ADAHELI. In: Proc. SPIE, Vol. 8148 of Society of Photo-Optical Instrumentation Engineers (SPIE) Conference Series, p. 81480U. https://doi.org/10.1117/12.893579. 
Stangalini M, Piazzesi R, Speziali R, Dal Sasso L. 2018. SAMM: The solar activity MOF monitor. In Proc. SPIE, Vol. 10700 of Society of Photo-Optical Instrumentation Engineers (SPIE) Conference Series, p. 107001K. https://doi.org/10.1117/12.2313373.

Supriya HD, Smitha HN, Nagendra KN, Stenflo JO, Bianda M, Ramelli R, Ravindra B, Anusha LS. 2014. Center-to-limb observations and modeling of the Ca I $4227 \AA$ line. Astrophys $J$ 793(1): 42. https://doi.org/10.1088/0004-637X/793/1/42.

Tomczyk S, Cacciani A, Veitzer SA. 1993, LOWL - an instrument to observe low-degree solar oscillations. In: GONG 1992. Seismic Investigation of the Sun and Stars, Brown TM (Ed.), Vol. 42 of Astronomical Society of the Pacific Conference Series, p. 469.

Tomczyk S, Streander K, Card G, Elmore D, Hull H, Cacciani A. 1995. An instrument to observe low-degree solar oscillations. Sol Phys 159(1): 1-21. https://doi.org/10.1007/BF00733027.

Uitenbroek H. 2006. Chromospheric diagnostics. In: Solar MHD theory and observations: A high spatial resolution perspective, Leibacher J, Stein RF, Uitenbroek H (Eds.), Vol. 354 of Astronomical Society of the Pacific Conference Series, p. 313.

Veronig AM, Pötzi W. 2016. Ground-based observations of the solar sources of space weather. In: Coimbra Solar Physics Meeting: Ground-based Solar Observations in the Space Instrumentation Era, Dorotovic I, Fischer CE, Temmer M (Eds.), Vol. 504 of Astronomical Society of the Pacific Conference Series, p. 247.

Wallace L, Hinkle KH, Livingston WC, Davis SP. 2011. An optical and near-infrared (2958-9250 ̊) solar flux Atlas. Astrophys $J$ Suppl Ser 195(1): 6. https://doi.org/10.1088/0067-0049/195/1/6.

Wang Y, Liu J, Jiang Y, Erdélyi R. 2019. CME arrival time prediction using convolutional neural network. Astrophys $J$ 881(1): 15. https://doi.org/10.3847/1538-4357/ab2b3e.

Wang J, Zhang Y, Hess Webber SA, Liu S, Meng X, Wang T. 2020. Solar flare predictive features derived from polarity inversion line masks in active regions using an unsupervised machine learning algorithm. Astrophys J 892(2): 140. https://doi.org/10.3847/15384357/ab7b6c.
Welsch BT, Longcope DW. 2003. Magnetic helicity injection by horizontal flows in the Quiet Sun. I. Mutual-helicity flux. Astrophys J 588: 620-629. https://doi.org/10.1086/368408.

Wiegelmann T, Sakurai T. 2012. Solar force-free magnetic fields. Living Rev Sol Phys 9: Article ID 5. https://doi.org/10.12942/lrsp2012-5.

Xia Y, Cheng X, Li F, Yang Y, Lin X, Jiao J, Du L, Wang J, Yang G. 2020. Sodium lidar observation over full diurnal cycles in Beijing, China. Appl Opt 59(6): 1529. https://doi.org/10.1364/ AO.382077.

Yan Y, Zhang J, Wang W, Liu F, Chen Z, Ji G. 2009. The Chinese Spectral Radioheliograph - CSRH. Earth Moon Planets 104(1-4): 97-100. https://doi.org/10.1007/s11038-008-9254-y.

Yan Y, Wang W, Chen L, Liu F, Geng L, Chen Z. 2018. New interplanetary scintillation array in China for space weather. Sun Geosph 13: 153-155. https://doi.org/10.31401/SunGeo.2018.02.05.

Yan Y, Chen Z, Wang W, Liu F, Geng L, Chen L, Tan C, Chen X, Su C, Tan B. 2021. Mingantu spectral radioheliograph for solar and space weather studies. Front Astron Space Sci 8(584043): 1-13. https://doi.org/10.3389/fspas.2021.584043, https://www. frontiersin.org/articles/10.3389/fspas.2021.584043/abstract.

Yang Y, Cheng X, Li F, Hu X, Lin X, Gong S. 2011. A flat spectral Faraday filter for sodium lidar. Opt Lett 36(7): 1302. https://doi. org/10.1364/OL.36.001302.

Ye Y, Korsós MB, Erdélyi R. 2018. Detailed analysis of dynamic evolution of three Active Regions at the photospheric level before flare and CME occurrence. Adv Space Res 61(2): 673-682. https://doi.org/10.1016/j.asr.2017.09.038.

Zhao J, Hing D, Chen R, Hess Webber S. 2019. Imaging the Sun's far-side active regions by applying multiple measurement schemes on multiskip acoustic waves. Astrophys $J$ 887(2): 216 . https://doi. org/10.3847/1538-4357/ab5951.

Zielińska JA, Beduini FA, Lucivero VG, Mitchell MW. 2014. Atomic filtering for hybrid continuous-variable/discrete-variable quantum optics. Opt Express 22(21): 25307-25317. https://doi. org/10.1364/OE.22.025307.

Cite this article as: Erdélyi R, Korsós MB, Huang X, Yang Y, Pizzey D, et al. 2022. The Solar Activity Monitor Network - SAMNet. J. Space Weather Space Clim. 12, 2. https://doi.org/10.1051/swsc/2021025. 